\title{
Correlation functions of three heavy operators - the AdS contribution
}

\author{
Romuald A. Janik and Andrzej Wereszczyński \\ Institute of Physics, Jagiellonian University, \\ ul. Reymonta 4, 30-059 Kraków, Poland \\ E-mail: romuald@th.if.uj.edu.pl, wereszcz@th.if.uj.edu.pl
}

ABSTRACT: We consider operators in $\mathcal{N}=4$ SYM theory which are dual, at strong coupling, to classical strings rotating in $S^{5}$. Three point correlation functions of such operators factorize into a universal contribution coming from the AdS part of the string sigma model and a state-dependent $S^{5}$ contribution. Consequently a similar factorization arises for the OPE coefficients. In this paper we evaluate the AdS universal factor of the OPE coefficients which is explicitly expressed just in terms of the anomalous dimensions of the three operators.

KEYWORDS: Supersymmetric gauge theory, AdS-CFT Correspondence, Integrable Field Theories

ARXIV EPRINT: 1109.6262 


\section{Contents}

1 Introduction 1

2 Two-point correlation functions $\quad 3$

3 Three-point correlation functions - general features 5

4 Our strategy 6

5 Pohlmeyer reduction $\quad 8$

$\begin{array}{llr}5.1 \text { Overlaps } & 12\end{array}$

$\begin{array}{lll}5.2 & \text { Reconstruction formulas } & 16\end{array}$

6 The AdS action 18

7 The regularized Pohlmeyer contribution $\quad 18$

$\begin{array}{ll}7.1 \text { Comparison with numerics } & 23\end{array}$

8 The regularized divergent contribution $\quad 24$

9 The final AdS contribution to the OPE coefficients 26

$\begin{array}{lr}10 \text { Summary and outlook } & 29\end{array}$

$\begin{array}{ll}\text { A Details of the numerical computation } & 31\end{array}$

B Evaluation of the integrals $\quad 32$

B.1 $\int_{C_{+}}\left(\int_{P_{+}}^{P} \omega\right) \eta=-i \pi \frac{1}{6} \quad 32$

B.2 $\int_{C_{ \pm}} \eta=0 \quad 32$

$\begin{array}{ll}\text { C The zero mode parts } & 33\end{array}$

D Small $a$ asymptotics of $\tilde{h}(a) \quad 34$

\section{Introduction}

In recent years there has been huge progress in exactly solving the $\mathcal{N}=4$ Super-Yang-Mills theory in the planar limit [1]-[15] . This is particularly remarkable as $\mathcal{N}=4 \mathrm{SYM}$ is an interacting four-dimensional gauge theory with highly nontrivial dynamics. All previous examples of exact solvability in Quantum Field Theories were restricted either to two dimensional theories or, in the case of higher dimensional theories, to some supersymmetric subsector or to theories with much simpler dynamics like topological field theories. 
The solvability of $\mathcal{N}=4 \mathrm{SYM}$ arises due to the AdS/CFT correspondence [16-18], according to which the gauge theory is equivalent to string theory in $\mathrm{AdS}_{5} \times S^{5}$. Therefore $\mathcal{N}=4$ SYM avoids the no-go theorems for integrable field theories in more than twodimensions by translating its dynamics into properties of the two-dimensional worldsheet QFT of the superstring in $\mathrm{AdS}_{5} \times S^{5}$ which is an integrable QFT.

Since $\mathcal{N}=4 \mathrm{SYM}$ is a conformal field theory, all correlation functions of local operators are, in principle, determined by a much smaller set of data: the set of conformal dimensions (equivalently anomalous dimensions of gauge theory operators) and the OPE coefficients. These data can be extracted from the knowledge of 2- and 3-point correlation functions.

In the AdS/CFT case, however, the anomalous dimensions are extracted not from 2-point correlation functions but directly as eigenvalues of the dilatation operator, which translates to energies of string states in $\mathrm{AdS}_{5} \times S^{5}$. Therefore the problem of finding all anomalous dimensions reduces to finding the energy levels of the two-dimensional worldsheet QFT on a cylinder. Currently we have a very complete understanding of the spectrum of conformal dimensions which is described by a set of Thermodynamic Bethe Ansatz equations [11]-[15]. The methods used are similar to the ones employed for relativistic integrable field theories, although their generalization to the AdS/CFT case is far from trivial due to many unique features of the worldsheet QFT.

For the case of OPE coefficients, however, there seems to be no alternative to a direct computation of 3-point correlation functions. It is convenient to classify the operators into three main groups, depending on their behaviour at strong coupling: 'light', 'medium' and 'heavy' operators. The 'light' operators are BPS and dual to supergravity fields. Consequently their anomalous dimensions do not depend on the coupling. The next class of operators are the lightest massive string states whose dimensions scale like $\lambda^{\frac{1}{4}}$. A classical example of these 'medium' operators is the Konishi operator. Finally, the 'heavy' operators have large charges (of the order of $\lambda^{\frac{1}{2}}$ ) and are dual to classical string states with anomalous dimensions scaling like $\lambda^{\frac{1}{2}}[19]-[21]$. Although very useful, this is only a rough and nonexhaustive classification. BPS operators with large charges may for all practical purposes behave like 'heavy' operators. There may be operators with dimensions like $\lambda^{\frac{1}{2}}$ which may be very quantum and without a classical string description (like BPS operators with two large charges).

The techniques for computing 3-point correlation functions are very well developed for the case of 'light' operators, i.e. BPS operators dual to supergravity fields [22]-[23]. Unfortunately these OPE coefficients are protected and do not depend on gauge theory coupling.

For unprotected operators, the techniques for computing even 2-point correlation functions have been developed only recently [24] (but see also [25] and [26]). These results have been used to compute OPE coefficients between two 'heavy' and one 'light' operator using the known classical solution corresponding to a 2-point function and integrating a supergravity propagator over the classical string worldsheet [27]-[40]. It has been further extended for correlators involving two Wilson loops and a 'light' operator [41, 42].

An intermediate case recently considered in the literature involved a geodesic approximation for the three operators [43]. Such an approximation may be very relevant to the case of three 'medium' operators which are not sufficiently heavy to generate an extended, non-pointlike, surface. 
The goal of this paper is to compute (the AdS part of) the 3-point correlation functions of three 'heavy' operators. We assume that these operators do not have any spin in $\mathrm{AdS}_{5}$. The main difficulty lies in the fact that a novel type of a classical solution has to be found. Moreover, in contrast to the spectral problem, there is no analog of this problem for conventional relativistic integrable field theories, therefore we do not have any guide in this respect.

The computation of the OPE coefficients for three 'heavy' operators is especially interesting in view of the fundamental importance that the integrable classification of finite-gap spinning string solutions and their comparison with 1-loop Bethe Ansatz results had in arriving at the all-loop interpolation. We hope that a similar comparison with weak coupling data $[44,45]$ will be very illuminating also in the case of OPE coefficients.

The plan of this paper is as follows. In section 2, we briefly review the case of 2-point correlation functions, and in section 3 the general features of the problem of finding 3-point correlation functions. In section 4 , we give an overview of our approach to this problem, in order for the reader to not get lost in the technicalities. In section 5, we review Pohlmeyer reduction, and give our main technical results necessary for later computations - we solve functional equations for the products between the solutions of the linear system on the 3punctured sphere and give formulas for reconstructing the classical solution in $\mathrm{AdS}_{2}$ from the Pohlmeyer data. We then proceed, using these results, to evaluate in the next 3 sections the two main parts of the AdS contribution to the correlation function. We give our final result in section 9 and discuss the limits of small and large anomalous dimensions and the link of the latter to the Painlevé III transcendent. We close the paper with a discussion and several appendices with some technical details.

\section{Two-point correlation functions}

In this section we will briefly review the computation of 2-point correlation functions for the class of operators that we are considering in this paper, namely operators corresponding to classical string solutions with no charges in AdS [19]-[21].

The approach introduced in [24], amounts to computing a cylinder amplitude (with additional wavefunctions included in order to project on the specific string state that we are interested in) with the boundary conditions such that the string worldsheet approaches two given points on the boundary regularized by a cut-off $z=\mathcal{E}$. This is done by a classical computation, where the corresponding solution is just a geodesic in the AdS part and in the $S^{5}$ part coincides with the unmodified $S^{5}$ spinning string solution used in the conventional calculation of the anomalous dimension. Then one performs a saddle point evaluation of the integral over the modular parameter. The outcome is ${ }^{1}$ that the saddle point value of the modular parameter is purely imaginary thus effectively making the worldsheet Euclidean.

This generic pattern indicates that we could have started directly at the saddle point, with the worldsheet having the topology of a 2-punctured sphere (again with small disks corresponding to $z=\mathcal{E}$ cut out), and the Euclidean solution satisfying Virasoro constraints.

\footnotetext{
${ }^{1}$ See specific examples in [24].
} 
The Euclidean solution for the operators in question has the following simple structure. The $\mathrm{AdS}_{5}$ part reduces just to a geodesic in the $\mathrm{AdS}_{2}$ subspace which contains the two gauge theory operator insertion points on the boundary. Explicitly we have

$$
x=\frac{R}{2} \tanh \kappa \tau+x_{0} \quad z=\frac{R}{2} \frac{1}{\cosh \kappa \tau}
$$

where the distance between the operator insertion points is $x_{1}-x_{2}=R$. Imposing the target space cut-off $z=\mathcal{E}$ translates into a worldsheet cut-off which limits the range of $\tau$ to

$$
\Delta \tau=\frac{2}{\kappa} \log \frac{R}{\mathcal{E}}
$$

The $S^{5}$ part is just the Wick rotated spinning string solution, a simple example being a circular string with equal angular momenta given in terms of the standard angular coordinates on $S^{3} \subset S^{5}$ by

$$
\psi=\sigma \quad \phi_{1}=\phi_{2}=i \omega \tau
$$

One should note that due to the $i$, the solution is inherently complex and in fact does not look like any kind of spinning string. Virasoro constraints link $\kappa$ and $\omega$ through $\kappa=\sqrt{1+\omega^{2}}$. In general we have $\kappa=\Delta$, where $\Delta$ is the dimension of the operator.

The 2-point correlation function is now obtained by i) evaluating the $\mathrm{AdS}_{2}$ action on the AdS geodesic part and ii) evaluating the $S^{5}$ action together with wavefunction contributions, which transforms the action integral into an Euclidean energy integral.

Explicitly we get

$$
\exp \underbrace{\left\{-\sqrt{\lambda} \sqrt{1+\omega^{2}} \log \frac{R}{\mathcal{E}}\right\}}_{\text {AdS action }} \cdot \exp \underbrace{\left\{-\sqrt{\lambda} \sqrt{1+\omega^{2}} \log \frac{R}{\mathcal{E}}\right\}}_{S^{5} \text { energy }}
$$

which reproduces the 2-point correlation function with the correct value of the anomalous dimension

$$
\left\langle O\left(x_{1}\right) O\left(x_{2}\right)\right\rangle=\left(\frac{\mathcal{E}}{R}\right)^{2 \sqrt{\lambda} \sqrt{1+\omega^{2}}}
$$

The wavefunction factors in the $S^{5}$ part are crucial for the correct answer. The Virasoro constraint sets the only free parameter $\kappa$ in the $\mathrm{AdS}_{2}$ geodesic solution (2.1) to be equal to $\Delta$, the (anomalous) dimension of the corresponding operator. ${ }^{2}$ Therefore, the AdS part of the solution is completely determined just by the dimension of the operator in question and not by any further details of the specific operator. We will see that the same property will hold also for 3-point correlation functions. In the following we will use complex coordinates which, in the present case, read

$$
w=e^{\tau+i \sigma} \quad \bar{w}=e^{\tau-i \sigma}
$$

putting the two punctures at $w=0$ and $w=\infty$.

\footnotetext{
${ }^{2}$ More precisely the dimension $\Delta$ is $\Delta=\sqrt{\lambda} \Delta$.
} 
Finally, let us note that the target space cut-off $\mathcal{E}$ enters (2.5) essentially as the normalization of our operators. This is important as in the case of 3-point correlation functions we have to retain exactly the same normalization of operators as for 2-point functions in order to extract unambiguosly the OPE coefficients. This leads to severe difficulties, such as linking the worldsheet cut-offs around the 3 punctures to the single target space cut-off $z=\mathcal{E}$. This is highly nontrivial due to the lack of an explicit classical AdS solution in the case of 3-point correlation functions. If one would adopt a different approach ${ }^{3}$ of using vertex operators $[26,46,47]$, then the difficulties remain but appear in different places. In the vertex operator approach, one computes the worlsheet integral over the whole punctured sphere with the vertex operator contributions sitting directly at the punctures. For 2-point functions one then just neglects infinities. For 3-point functions it is not clear how to control possible finite renormalizations. It would be very interesting to understand quantitatively the precise dictionary between the two approaches.

\section{Three-point correlation functions - general features}

In order to compute the 3-point correlation function of heavy operators, we have to find a classical solution of string equations of motion in Euclidean signature with the topology of a sphere with 3 punctures, with the property that the solution close to each puncture associated with a given gauge theory operator $O_{k}$ looks asymptotically like a solution corresponding to a 2-point correlation function of the operator $O_{k}$.

The classical bosonic equations of motion in $\mathrm{AdS}_{5} \times S^{5}$ reduce to two independent sets of equations, one on $S^{5}$, the other on $\mathrm{AdS}_{5}$, which are coupled together only through the Virasoro constraint

$$
T_{\mathrm{AdS}_{5}}(w)+T_{S^{5}}(w)=0
$$

where $w$ is the holomorphic worldsheet coordinate, and $T_{\mathrm{AdS}_{5}}(w)$ (resp. $\left.T_{S^{5}}\right)$ is the classical energy-momentum tensor of the $\mathrm{AdS}_{5}\left(\right.$ resp. $S^{5}$ ) part of the $\sigma$-model.

For operators which do not have any spins, the $\mathrm{AdS}_{5}$ part of the problem greatly simplifies. Then, without loss of generality, we put the gauge theory operator insertion points of all three operators on a single line. Consequently, the $\mathrm{AdS}_{5}$ part of the string solution is contained in an (Euclidean) $\mathrm{AdS}_{2}$ subspace. The problem, however, does not trivialize as we are not looking for a minimal surface but have a prescribed nonzero energymomentum tensor $T_{\mathrm{AdS}_{2}}(w)$.

Fortunately, the $\mathrm{AdS}_{2}$ energy-momentum tensor can be explicitly expressed just in terms of the anomalous dimensions of the three operators entering the 3-point correlation function. From now on we will denote $T_{\mathrm{AdS}_{2}}(w)$ by $T(w)$.

In order to find the explicit form of $T(w)$, recall that the classical solution should approach, at the punctures, 2-point solutions which are explicitly known. In particular $T(w)$ for the 2-point solutions is given by

$$
T_{2 \text {-point }}(w)=\frac{\Delta^{2} / 4}{w^{2}}
$$

\footnotetext{
${ }^{3}$ Which should of course be in the end equivalent.
} 
Therefore at the punctures $T(w)$ should have at most poles of $2^{\text {nd }}$ order with the leading coefficients $\Delta_{k}^{2} / 4$ determined by the dimension of the operator inserted at $w=w_{k}$. Since $T(w)$ is holomorphic and transforms under inversion like a $(2,0)$ tensor

$$
T(w) \rightarrow \frac{1}{u^{4}} T\left(\frac{1}{u}\right)
$$

its form is uniquely determined. Without loss of generality we may put the punctures at $w= \pm 1$ and $w=\infty$. Then, for the case of equal conformal weights $\Delta$ at $w= \pm 1$ and $\Delta_{\infty}$ at $w=\infty, T(w)$ is given by

$$
T(w)=\frac{\Delta_{\infty}^{2}}{4} \frac{w^{2}+a^{2}}{\left(1-w^{2}\right)^{2}} \quad \text { where } \quad a^{2}=\frac{4 \Delta^{2}}{\Delta_{\infty}^{2}}-1
$$

In the present paper for simplicity we will predominantly consider the above symmetric case.

To summarize, we thus have to evaluate the action

$$
\exp \left(-\frac{\sqrt{\lambda}}{\pi} \int_{\Sigma} \mathcal{L}_{\text {AdS }_{2}}^{\text {Polyakov }} d^{2} w\right) \equiv\left(-\frac{\sqrt{\lambda}}{\pi} \int_{\Sigma} \frac{\partial z \bar{\partial} z+\partial x \bar{\partial} x}{z^{2}} d^{2} w\right)
$$

for a classical solution approaching the operator insertion points $x_{k}$ at $w=-1,1, \infty$ subject to the constraint

$$
\frac{(\partial z)^{2}+(\partial x)^{2}}{z^{2}}=T(w)
$$

\section{Our strategy}

As described in the previous section, the 3-point correlation functions for three heavy operators with no spins in $\mathrm{AdS}_{5}$ factorize into a product of an $S^{5}$ and an $\mathrm{AdS}_{2}$ contribution evaluated for a worldsheet with the topology of a 3-punctured sphere. Similarly as for 2point functions we regularize the worldsheet by cutting out small disks of radii $\varepsilon_{i}$ around the punctures which are defined by the condition that on their boundaries

$$
z=\mathcal{E}
$$

where $z$ is the AdS radial coordinate in the Poincare patch ( $z=0$ is the AdS boundary). $\mathcal{E}$ is the target space cut-off which is taken to be very small. It is necessary to ensure that $z=\mathcal{E}$ around each puncture in order to have the same normalization of operators in 2and 3-point correlation functions so as to unambigously extract the OPE coefficients.

For the $\mathrm{AdS}_{2}$ part, we have to evaluate the action of the classical solution, while for the $S^{5}$ part we have to include, in addition, contributions from the classical wavefunctions of the external states. Therefore, the 3 -point correlation function is schematically given by

$$
e^{-\frac{\sqrt{\lambda}}{\pi} \int_{\Sigma \backslash\left\{\varepsilon_{i}\right\}} \mathcal{L}_{\mathrm{AdS}_{2}}^{\text {Polyakov }}} \cdot \underbrace{\Psi_{1} \Psi_{2} \Psi_{3}^{*} e^{-\frac{\sqrt{\lambda}}{\pi} \int_{\Sigma \backslash\left\{\varepsilon_{i}\right\}} \mathcal{L}_{S^{5}}^{\text {Polyakov }}}}_{e^{-\frac{\sqrt{\lambda}}{\pi} \int_{\Sigma \backslash\left\{\varepsilon_{i}\right\}} S^{5} \text { contribution }}}
$$


Since both exponents have logarithmic divergences around the punctures, it is convenient to subtract and add $\sqrt{T(w) \bar{T}(\bar{w})}$ regularizing the integrals. This yields

$$
e^{-\frac{\sqrt{\lambda}}{\pi} \int_{\Sigma}\left(\mathcal{L}_{\text {AdS }_{2}}^{\text {Polyakov }}-\sqrt{T \bar{T}}\right)-\frac{\sqrt{\lambda}}{\pi} \int_{\Sigma \backslash\left\{\varepsilon_{i}\right\}} \sqrt{T \bar{T}}}
$$

for the $\mathrm{AdS}_{2}$ part and

$$
e^{-\frac{\sqrt{\lambda}}{\pi} \int_{\Sigma}\left(S^{5} \text { contribution }-\sqrt{T \bar{T}}\right)-\frac{\sqrt{\lambda}}{\pi} \int_{\Sigma \backslash\left\{\varepsilon_{i}\right\}} \sqrt{T \bar{T}}}
$$

for the $S^{5}$ part. The first terms in the above expressions are now finite and can be integrated over the whole punctured sphere, while the explicit dependence on the worldsheet cut-offs appears only in the second integral with a known integrand.

In this paper we will compute the contribution (4.3) together with the second term in (4.4), leaving the remaining factor

$$
e^{-\frac{\sqrt{\lambda}}{\pi} \int_{\Sigma}\left(S^{5} \text { contribution }-\sqrt{T \bar{T}}\right)}
$$

for further investigation.

In order to compute

$$
e^{-\frac{\sqrt{\lambda}}{\pi} \int_{\Sigma}\left(\mathcal{L}_{\mathrm{AdS}_{2}}^{\text {Polyakov }}-\sqrt{T \bar{T}}\right)}
$$

we will use Pohlmeyer reduction [48, 49] and adapt the methods of [50] to evaluate this expression. Firstly, one transforms the above integral into an integral of the wedge product of two closed 1-forms on a double cover of $\Sigma$. Secondly, one uses Riemann reciprocity (Riemann bilinear identity) to express the integral in terms of products of integrals of the 1-forms on certain open cycles. Thirdly, one links the above 1-form integrals to the asymptotics in the spectral parameter $(\xi \rightarrow 0)$ of appropriate skew products between specific solutions (associated with each puncture) of the Pohlmeyer linear system. Thus the evaluation of the integral (4.6) is reduced to the knowledge of appropriate skew products as a function of the spectral parameter.

The remaining integral

$$
e^{-\frac{\sqrt{\lambda}}{\pi} \int_{\Sigma \backslash\left\{\varepsilon_{i}\right\}} \sqrt{T \bar{T}}}
$$

can be evaluated analytically in the small $\varepsilon_{i}$ limit. The main difficulty lies in linking the worldsheet cut-offs $\left\{\varepsilon_{i}\right\}$ to the target space cut-off $z=\mathcal{E}$, without an explicit knowledge of the classical solution. To do that we need formulas for reconstructing the classical solution from Pohlmeyer data. Fortunately, since the classical solution should approach the known solutions for 2-point functions close to the punctures, we can get explicit formulas relating the positions of the gauge theory operator insertion points $x_{k}$ and the target space cut-off $\mathcal{E}$ to the worldsheet cut-offs $\left\{\varepsilon_{i}\right\}$ in terms of the skew products mentioned above, but this time evaluated at $\xi=1$. Using this knowledge, the (two copies of the) integral (4.7) yield the standard space-time dependent part of the 3-point CFT correlation function, as well as a finite contribution to the OPE coefficient expressed in terms of the skew products at $\xi=1$. 
The skew products between the specific solutions (of the Pohlmeyer linear system) associated to each puncture are therefore a key ingredient in the evaluation of the 3-point correlation function. We will often refer to these chosen solutions as 'elementary solutions'.

In the following section we introduce the main features of Pohlmeyer reduction, the elementary solutions associated with each puncture and define the skew products. Then we derive and solve functional equations for the skew products of the elementary solutions as a function of the spectral parameter $\xi$. Finally we state the reconstruction formulas which link the operator insertion points and the target space cut-off to appropriate skew products.

After this preparatory part, we proceed to evaluate the integral (4.6) in section 7 and the divergent contribution (4.7) in section 8. Then we put together the obtained formulas into the final AdS contribution to the OPE coefficient and analyze the limits of large and small anomalous dimensions as well as the extremal limit.

Before we end this overview, let us remark that the same decomposition (4.2) could also be performed for a 2-punctured sphere corresponding to a 2-point correlation function (of course in this case only two wavefunctions would appear). Then it turns out that both the 'nontrivial' parts (4.6) and (4.5) are identically zero. However it is interesting to note that they vanish for quite different reasons. The AdS part (4.6) vanishes because it is evaluated on a trivial classical solution - a point-like string moving along a geodesic. The corresponding Pohlmeyer function is just identically zero and consequently (4.6) vanishes. On the $S^{5}$, however, we deal with arbitrarily complicated finite-gap solutions of arbitrary genus, which would have a highly nontrivial Pohlmeyer description. Yet, the wavefunction contributions transform the classical action into an integral of the energy density (in an appropriate coordinate system) and the resulting $S^{5}$ contribution exactly cancels the integral of $\sqrt{T \bar{T}}$. It is tempting to speculate that a similar simplification may occur for the case of 3-point functions.

\section{Pohlmeyer reduction}

Contrary to the well known case of Pohlmeyer reduction for minimal surfaces in $\mathrm{AdS}_{3}[48-$ 50], we need to perform Pohlmeyer reduction for classical solutions in $\mathrm{AdS}_{2}$ but with a prescribed nonzero energy-momentum tensor. Thus the classical solutions in $\mathrm{AdS}_{2}$ are of course not minimal surfaces. On the other hand, the full string solution, which takes into account both $\mathrm{AdS}_{2}$ and $S^{5}$ contributions, is a minimal surface.

The Pohlmeyer reduction for this case amounts to defining $\tilde{\gamma}(w, \bar{w})$ through

$$
\frac{\partial x \bar{\partial} x+\partial z \bar{\partial} z}{z^{2}}=\sqrt{T \bar{T}} \cosh \tilde{\gamma}
$$

where $T$ is the energy-momentum tensor $T(w)$. Then $\tilde{\gamma}(w, \bar{w})$ satisfies a modified form of Sinh-Gordon equation

$$
\partial \bar{\partial} \tilde{\gamma}=\sqrt{T \bar{T}} \sinh \tilde{\gamma}
$$


The solution corresponding to a 2-point function is just $\tilde{\gamma}(w, \bar{w}) \equiv 0$. Consequently, the boundary conditions close to each puncture are

$$
\tilde{\gamma} \rightarrow 0
$$

For the case relevant to 3-point correlation functions, $T(w)$ has two zeroes, and thus the form of Pohlmeyer reduction given by (5.1) is inconvenient as it would imply that all first derivatives vanish

$$
\partial z=\bar{\partial} z=\partial x=\bar{\partial} x=0
$$

at the zeroes of $T(w)$. This would be a very nongeneric situation as each such single equation gives a codimension one subspace. Their intersection is generically empty. This is even the case for pointlike strings appearing in 2-point functions. Consequently we will assume, as is the case for polygonal Wilson loops, that the right hand side of (5.1) is everywhere nonzero. This implies that $\tilde{\gamma}$ has to have logarithmic singularities at the zeros of $T(w)$.

To avoid this drawback, it is convenient to redefine $\tilde{\gamma}$ through

$$
\tilde{\gamma}=2 \gamma-\frac{1}{2} \log T \bar{T}
$$

Now (5.1) takes the form

$$
\frac{\partial x \bar{\partial} x+\partial z \bar{\partial} z}{z^{2}}=\frac{1}{2}\left(e^{2 \gamma}+T \bar{T} e^{-2 \gamma}\right)
$$

which does not lead to any problem at the zeroes of $T(w)$. The equation of motion becomes

$$
\partial \bar{\partial} \gamma=\frac{1}{4}\left(e^{2 \gamma}-T \bar{T} e^{-2 \gamma}\right)
$$

This is virtually the same as the setup for Wilson loop [50, 51] but with the polynomial defining the polygonal Wilson loop substituted by $T(w)$. We will discuss the similarities and differences in more detail at the end of the present section.

It is well known that the modified sinh-Gordon model is integrable. It is easiest to verify by making a holomorphic change of worldsheet coordinates to map this model into ordinary sinh-Gordon. However, due to the rather complicated analytical structure of the resulting domain we will not use this mapping in the sequel.

Below we review the main features of the integrability of sinh-Gordon model which will be important for us later.

There exists a family of flat connections parametrized by the spectral parameter $\xi$. We will also use the parametrization

$$
\xi=e^{\theta}
$$

The flat connection $J=J_{w} d w+J_{\bar{w}} d \bar{w}$ has the following components

$$
J_{w}=\frac{1}{2}\left(\begin{array}{cc}
\partial \gamma & -\frac{1}{\xi} e^{\gamma} \\
-\frac{1}{\xi} e^{-\gamma} T & -\partial \gamma
\end{array}\right) \quad J_{\bar{w}}=\frac{1}{2}\left(\begin{array}{cc}
-\bar{\partial} \gamma & -\xi e^{-\gamma} \bar{T} \\
-\xi e^{\gamma} & \bar{\partial} \gamma
\end{array}\right)
$$


Flatness is equivalent to the compatibility of the associated linear system

$$
\partial \Psi+J_{w} \Psi=0 \quad \bar{\partial} \Psi+J_{\bar{w}} \Psi=0
$$

which in turn is equivalent to the equation of motion (5.7). Another useful decomposition of the flat connection is

$$
J=\frac{1}{\xi} \Phi_{w} d w+A+\xi \Phi_{\bar{w}} d \bar{w}
$$

using which we may write the string action as

$$
\frac{\partial x \bar{\partial} x+\partial z \bar{\partial} z}{z^{2}}=2 \operatorname{tr} \Phi_{w} \Phi_{\bar{w}}
$$

Certain specific solutions of the linear system (5.10) associated with each puncture will be of key importance in the following. Since close to the punctures

$$
T(w) \sim \frac{\Delta^{2} / 4}{w^{2}}
$$

and

$$
\gamma \sim \frac{1}{4} \log T(w) \bar{T}(\bar{w})
$$

we get two solutions, which close to the puncture behave like

$$
\tilde{\Psi}_{1}=w^{\frac{\Delta}{4 \xi}} \bar{w}^{\frac{\Delta}{4} \xi}\left(\begin{array}{l}
w^{\frac{1}{4}} \bar{w}^{-\frac{1}{4}} \\
w^{-\frac{1}{4}} \bar{w}^{\frac{1}{4}}
\end{array}\right) \quad \tilde{\Psi}_{2}=w^{-\frac{\Delta}{4 \xi}} \bar{w}^{-\frac{\Delta}{4} \xi}\left(\begin{array}{c}
w^{\frac{1}{4}} \bar{w}^{-\frac{1}{4}} \\
-w^{-\frac{1}{4}} \bar{w}^{\frac{1}{4}}
\end{array}\right)
$$

It is clear that these solutions have nontrivial monodromies $e^{ \pm i \tilde{p}(\xi)}$ around the puncture $w=0$ with

$$
\tilde{p}(\xi)=\Delta \frac{\pi}{2}\left(\xi-\frac{1}{\xi}\right)+\pi
$$

It is in fact convenient to get rid of the $\pi$ by a gauge transformation $\Psi=V \tilde{\Psi}$ with

$$
V=\left(\begin{array}{cc}
\left(\frac{\left(w-w_{1}\right)\left(w-w_{2}\right)\left(w-w_{3}\right)}{\left(\bar{w}-\bar{w}_{1}\right)\left(\bar{w}-\bar{w}_{2}\right)\left(\bar{w}-\bar{w}_{3}\right)}\right)^{-\frac{1}{4}} & 0 \\
0 & \left(\frac{\left(w-w_{1}\right)\left(w-w_{2}\right)\left(w-w_{3}\right)}{\left(\bar{w}-\bar{w}_{1}\right)\left(\bar{w}-\bar{w}_{2}\right)\left(\bar{w}-\bar{w}_{3}\right)}\right)^{\frac{1}{4}}
\end{array}\right)
$$

Then our final basis of solutions associated to the puncture at $w=w_{1}$ is

$$
\begin{aligned}
& \Psi_{1}=\frac{i}{\sqrt{2}}\left(w-w_{1}\right)^{\frac{\Delta}{4 \xi}}\left(\bar{w}-\bar{w}_{1}\right)^{\frac{\Delta}{4} \xi}\left(\begin{array}{c}
u_{1} \\
u_{1}^{-1}
\end{array}\right) \\
& \Psi_{\overline{1}}=\frac{i}{\sqrt{2}}\left(w-w_{1}\right)^{-\frac{\Delta}{4 \xi}}\left(\bar{w}-\bar{w}_{1}\right)^{-\frac{\Delta}{4} \xi}\left(\begin{array}{c}
u_{1} \\
-u_{1}^{-1}
\end{array}\right)
\end{aligned}
$$

where the constant $u_{1}$ is given by

$$
u_{1}=\frac{\left(\bar{w}_{12} \bar{w}_{13}\right)^{\frac{1}{4}}}{\left(w_{12} w_{13}\right)^{\frac{1}{4}}}
$$


with $w_{i j}=w_{i}-w_{j}$. The solutions 1 (i.e. $\Psi_{1}$ ) and $\overline{1}$ (i.e. $\Psi_{\overline{1}}$ ) have the monodromies $e^{i p(\xi)}$ and $e^{-i p(\xi)}$ with the pseudomomentum given by

$$
p(\xi)=\Delta \frac{\pi}{2}\left(\xi-\frac{1}{\xi}\right) \quad(\equiv \Delta \pi \sinh \theta)
$$

Several comments are in order here. These solutions can be continued to the neighborhoods of the other punctures. Of course we do not know their analytical expressions so we cannot perform this explicitly. Generically these solutions will no longer be eigenstates of the monodromy operator around other punctures. However, since the space of solution of the linear system is two-dimensional, we can express 1 and $\overline{1}$ as linear combinations ${ }^{4}$ of an analogous basis $k$ and $\bar{k}$ at the puncture $w=w_{k}$. It is exactly these coefficients which are the key ingredients for the computation of the AdS part of the 3-point correlation function. In order to fix an inherent ambiguity associated with nontrivial monodromy, we have to fix once and for all the path of analytical continuation, whose detailed form will not be important for us.

It is clear that the pseudomomentum of the elementary solutions obeys the important general property

$$
p\left(e^{i \pi} \xi\right)=-p(\xi)
$$

This suggests that it should be possible to obtain the second solution $\overline{1}$ from the first 1 . Since just changing $\xi \rightarrow e^{i \pi} \xi$ modifies the expressions for the flat connection, one has to perform in addition a similarity transformation

$$
U J_{w, \bar{w}}(w, \bar{w} ; \xi) U^{-1}=J_{w, \bar{w}}\left(w, \bar{w} ; e^{i \pi} \xi\right)
$$

with $U=i \sigma_{3}$ to compensate. Therefore the second solution can be obtained from the first $\Psi(w, \bar{w} ; \xi)$ through

$$
\Psi_{\bar{k}}(w, \bar{w} ; \xi)=\sigma_{3} \Psi_{k}\left(w, \bar{w} ; e^{i \pi} \xi\right)
$$

This is a crucial property which allows for the formulation of a set of functional equations for the overlap coefficients.

Let us close this section with a comparison of the present set-up of a 3-point correlation function in $\mathrm{AdS}_{2}$ with the case of Pohlmeyer reduction for polygonal Wilson loops in $\mathrm{AdS}_{3}$.

In both cases we have the same modified sinh-Gordon model, but with the modification defined in terms of functions with quite different analytical properties. In the case of polygonal Wilson loops we have a polynomial with a single asymptotic region (covered by several Stokes sectors), here we have a rational function with three ( $2^{\text {nd }}$ order) poles and thus we have three distinct asymptotic regions. In the Wilson loop case, only the 'small' solution was unambigously defined, while here two solutions are uniquely specified as eigenfunctions of the monodromy operator at each puncture. Finally, the spacetime picture is quite different. In the Wilson loop case, the target-space was $\mathrm{AdS}_{3}$ and one had natural 'left-' and 'right-' linear problems. Here the target space is one dimension less $\left(\mathrm{AdS}_{2}\right)$ and we have to develop appropriate reconstruction formulas and impose boundary conditions characteristic of a 3-point correlation function (i.e. fixing the boundary coordinates of the operator insertion points $x_{k}$ and the target-space cut-off $z=\mathcal{E}$ ).

\footnotetext{
${ }^{4}$ With coefficients depending just on the spectral parameter.
} 


\subsection{Overlaps}

In this section we will derive and solve functional equations for the overlaps between the elementary solutions associated with each puncture defined in the previous section. For any two solutions of the linear system (5.10) $\Psi_{k}$ and $\Psi_{l}$, one defines the antisymmetric product (skew-product)

$$
\langle k l\rangle
$$

which is the determinant of the matrix formed by the column vectors $\Psi_{k}$ and $\Psi_{l}$. It is a function of the spectral parameter $\xi$ (or equivalently $\theta$ ). Our elementary solutions (5.18)(5.19) have the canonical normalization

$$
\langle k \bar{k}\rangle=1
$$

A characteristic feature of the product $\langle k l\rangle$ is that for any four solutions the relevant products obey a purely algebraic relation called the Schouten identity:

$$
\langle i j\rangle\langle k l\rangle+\langle i l\rangle\langle j k\rangle+\langle i k\rangle\langle l j\rangle=0
$$

In our case we have 6 distinguished solutions of the linear system $-1, \overline{1}, 2, \overline{2}, 3, \overline{3}$. Our aim is to find the skew-products between these solutions as functions of $\theta$, given the set of conformal weights $\Delta_{1}, \Delta_{2}$ and $\Delta_{3}$.

It is convienient to repackage the products between the various solutions into connection matrices $M_{k l}$ which transform the coordinates of a solution in the basis associated to the puncture $l$ to the coordinates in the basis associated to the puncture $k$.

The equation

$$
\left(\begin{array}{l}
\gamma \\
\delta
\end{array}\right)=\underbrace{\left(\begin{array}{ll}
A & B \\
C & D
\end{array}\right)}_{M_{k l}}\left(\begin{array}{l}
\alpha \\
\beta
\end{array}\right)
$$

amounts to the following equality between the elementary solutions

$$
\gamma \Psi_{k}+\delta \Psi_{\bar{k}}=\alpha \Psi_{l}+\beta \Psi_{\bar{l}}
$$

This means that

$$
\begin{aligned}
& \Psi_{l}=A \Psi_{k}+C \Psi_{\bar{k}} \\
& \Psi_{\bar{l}}=B \Psi_{k}+D \Psi_{\bar{k}}
\end{aligned}
$$

Now taking appropriate products gives an expression for $M_{k l}$ in terms of our fundamental products.

$$
M_{k l}=\left(\begin{array}{cc}
-\langle\bar{k} l\rangle & -\langle\bar{k} l\rangle \\
\langle k l\rangle & \langle k \bar{l}\rangle
\end{array}\right)
$$

The obvious compatibility conditions between the connection matrices

$$
M_{k m}=M_{k l} M_{l m} \quad M_{k l} M_{l k}=i d
$$


are in fact equivalent to the full set of Schouten identities. This can be easily seen by considering various choices for the solutions entering Schouten identities and comparing with appropriate elements of the matrix products (5.33).

The full set of functional relations for the products $\langle k l\rangle$ thus comprises the compatibility conditions (5.33) and the vanishing of total monodromy

$$
\Omega_{1} M_{13} \Omega_{3} M_{32} \Omega_{2} M_{21}=i d
$$

where

$$
\Omega_{k}=\left(\begin{array}{cc}
e^{i p_{k}(\theta)} & 0 \\
0 & e^{-i p_{k}(\theta)}
\end{array}\right)
$$

As they stand, the equations (5.33) and (5.34) are a complicated set of constraints for 12 unknown products. The key property which allows us to transform them into a set of solvable functional equations is the property (5.24). Using this construction we may relate 6 of the 12 unknown products to the other 6 but evaluated at a shifted value of the spectral parameter. Explicitly suppose that the $k$ elementary solution (eigenvector of the monodromy matrix around the puncture $w_{k}$ with the eigenvalue $\left.e^{i p_{k}}\right)$ is

$$
\Psi_{k}(w, \bar{w} ; \xi)=\left(\begin{array}{l}
a_{k} \\
b_{k}
\end{array}\right)
$$

Then the second solution $\bar{k}$ (with eigenvalue $e^{-i p_{k}}$ ) is obtained through

$$
\Psi_{\bar{k}}(w, \bar{w} ; \xi)=\sigma_{3} \Psi_{k}\left(w, \bar{w} ; \xi e^{i \pi}\right) \equiv\left(\begin{array}{c}
a_{k}^{++} \\
-b_{k}^{++}
\end{array}\right)
$$

where the superscript ' + ' denotes the shift $\theta \rightarrow \theta+i \pi / 2$. Using the fact ${ }^{5}$ that $a_{k}^{++++}=a_{k}$ and $b_{k}^{++++}=b_{k}$, it follows that

$$
\begin{aligned}
& \langle\bar{k} l\rangle=-\langle k l\rangle^{++} \\
& \langle\bar{k} l\rangle=-\langle k \bar{l}\rangle^{++}
\end{aligned}
$$

Now the relations (5.33) and (5.34) become functional equations for just 6 products.

Solution of the functional relation. In this section we will solve the full set of functional equations (5.33) and (5.34). Let us first define the three functions

$$
\begin{aligned}
& X_{32} \equiv\langle 32\rangle\langle 32\rangle^{++} \\
& X_{3 \overline{2}} \equiv\langle 3 \overline{2}\rangle\langle 3 \overline{2}\rangle^{++} \\
& X_{2 \overline{1}} \equiv\langle 2 \overline{1}\rangle\langle 2 \overline{1}\rangle^{++}
\end{aligned}
$$

Once we determine them explicitly, the products $\langle 32\rangle,\langle 3 \overline{2}\rangle$ and $\langle 2 \overline{1}\rangle$ will be expressed through convolution with a cosh kernel and zero-mode parts. The remaining products

\footnotetext{
${ }^{5}$ This follows from $\Psi_{k}^{++++}=\lambda_{k} \Psi_{k}$ and an argument that $\lambda_{k}=1$.
} 
$\langle 21\rangle,\langle 31\rangle$ and $\langle 3 \overline{1}\rangle$ will turn out to be expressed in terms of the first three and the pseudomomenta. In fact for our applications, it suffices to know the formulas for the products between the unbarred solutions: $\langle 12\rangle,\langle 23\rangle$ and $\langle 31\rangle$ - so all of them will be expressed through $X_{32}$ and some permutation of indices. However in this section, for completeness, we will solve all equations.

We start from the equation $M_{32} M_{21}=M_{31}$. This just expresses $\langle 31\rangle$ and $\langle 3 \overline{1}\rangle$ through the Schouten identities:

$$
\begin{aligned}
& \langle 31\rangle=\langle 32\rangle\langle 2 \overline{1}\rangle^{++}+\langle 21\rangle\langle 3 \overline{2}\rangle \\
& \langle 3 \overline{1}\rangle=\langle 32\rangle\langle 21\rangle^{++}+\langle 2 \overline{1}\rangle\langle 3 \overline{2}\rangle
\end{aligned}
$$

Then define $Y_{1}$ and $Y_{3}$ as

$$
\begin{aligned}
Y_{1} & =\frac{\langle 3 \overline{2}\rangle\langle 21\rangle}{\langle 31\rangle} \\
Y_{3} & =\frac{\langle 3 \overline{2}\rangle\langle 2 \overline{1}\rangle}{\langle 3 \overline{1}\rangle}
\end{aligned}
$$

We can express $\langle 21\rangle$ in terms of $Y_{1}$. Plugging the results into the formula for $Y_{3}$ we see that we can express $X_{3 \overline{2}}$ as

$$
X_{3 \overline{2}}=X_{32} \frac{Y_{1}^{++} Y_{3}}{\left(1-Y_{1}^{++}\right)\left(1-Y_{3}\right)}
$$

At this stage is convenient to rewrite the monodromy equation (5.34) in the form

$$
M_{32} \Omega_{2} M_{21}=\Omega_{3}^{-1} M_{31} \Omega_{1}^{-1}
$$

The entries of (5.48) are in fact the counterparts of the $\bar{Y}$ functions introduced by Maldacena and Zhiboedov ([52] figure 5). obtain

The equation (5.48) enables us to determine $Y_{1}$ and $Y_{3}$ defined earlier. Explicitly we

$$
\begin{aligned}
& Y_{1}=\frac{1-e^{i\left(p_{3}-p_{1}-p_{2}\right)}}{1-e^{-2 i p_{2}}} \\
& Y_{3}=\frac{1-e^{i\left(p_{3}+p_{1}-p_{2}\right)}}{1-e^{-2 i p_{2}}}
\end{aligned}
$$

Now we proceed to the equation $M_{21} M_{13}=M_{23}$ which enables us to determine $X_{2 \overline{1}}$, and finally the equation $M_{13} M_{32}=M_{12}$ determines $X_{32}$. We check that all the remaining compatibility conditions of (5.33) are satisfied.

Consequently, the final functional equations for $\langle 32\rangle,\langle 3 \overline{2}\rangle$ and $\langle 2 \overline{1}\rangle$ read

$$
\begin{aligned}
\langle 32\rangle\langle 32\rangle^{++} & =\frac{\sin \frac{p_{1}-p_{2}-p_{3}}{2} \sin \frac{p_{1}+p_{2}+p_{3}}{2}}{\sin p_{2} \sin p_{3}} \\
\langle 3 \overline{2}\rangle\langle 3 \overline{2}\rangle^{++} & =\frac{\sin \frac{p_{3}-p_{1}-p_{2}}{2} \sin \frac{p_{2}-p_{1}-p_{3}}{2}}{\sin p_{2} \sin p_{3}} \\
\langle 2 \overline{1}\rangle\langle 2 \overline{1}\rangle^{++} & =\frac{\sin \frac{p_{1}-p_{2}-p_{3}}{2} \sin \frac{p_{2}-p_{1}-p_{3}}{2}}{\sin p_{1} \sin p_{2}}
\end{aligned}
$$


with the right hand sides being exactly our functions $X_{32}, X_{3 \overline{2}}$ and $X_{2 \overline{1}}$. In the above expressions we did not use the specific form of $p_{k}(\theta)$ given by (5.21) but only the generic property

$$
p_{k}(\theta+i \pi)=-p_{k}(\theta)
$$

Therefore, the above solution may have a much greater range of applicability than the specific case of no spin in $\mathrm{AdS}_{5}$ that we consider in the present paper.

Let us now specialize to the pseudomomenta (5.21) and use the parametrization $\xi=e^{\theta}$. Then

$$
p_{k}(\theta)=\Delta_{k} \pi \sinh \theta
$$

The above functional equations can be recast in the form

$$
\begin{aligned}
\langle 32\rangle^{+}\langle 32\rangle^{-} & =-\frac{\sinh \left(\frac{\Delta_{2}+\Delta_{3}-\Delta_{1}}{2} \pi \cosh \theta\right) \sinh \left(\frac{\Delta_{1}+\Delta_{2}+\Delta_{3}}{2} \pi \cosh \theta\right)}{\sinh \left(\Delta_{2} \pi \cosh \theta\right) \sinh \left(\Delta_{3} \pi \cosh \theta\right)} \\
\langle 3 \overline{2}\rangle^{+}\langle 3 \overline{2}\rangle^{-} & =\frac{\sinh \left(\frac{\Delta_{1}+\Delta_{3}-\Delta_{2}}{2} \pi \cosh \theta\right) \sinh \left(\frac{\Delta_{1}+\Delta_{2}-\Delta_{3}}{2} \pi \cosh \theta\right)}{\sinh \left(\Delta_{2} \pi \cosh \theta\right) \sinh \left(\Delta_{3} \pi \cosh \theta\right)} \\
\langle 2 \overline{1}\rangle^{+}\langle 2 \overline{1}\rangle^{-} & =\frac{\sinh \left(\frac{\Delta_{2}+\Delta_{3}-\Delta_{1}}{2} \pi \cosh \theta\right) \sinh \left(\frac{\Delta_{1}+\Delta_{3}-\Delta_{2}}{2} \pi \cosh \theta\right)}{\sinh \left(\Delta_{1} \pi \cosh \theta\right) \sinh \left(\Delta_{2} \pi \cosh \theta\right)}
\end{aligned}
$$

As mentioned before, for our purposes we will be interested in the solution of the first equation. The formulas for $\langle 12\rangle$ and $\langle 31\rangle$ can then be obtained simply by a permutation of the $\Delta_{i}$ 's.

The right hand side of the first equation has the property that it approaches a constant when $\theta \rightarrow \pm \infty$ thus making the solution simpler. The basic functional equation to solve is

$$
f_{a}^{+} f_{a}^{-}=1-e^{-a \pi \cosh \theta}
$$

which can be solved by convolution

$$
f_{a}(\theta)=\exp \int_{-\infty}^{\infty} \frac{d \theta^{\prime}}{2 \pi} \frac{\log \left(1-e^{-a \pi \cosh \theta^{\prime}}\right)}{\cosh \left(\theta-\theta^{\prime}\right)}
$$

Therefore we get the following expression for the product $\langle 32\rangle$ :

$$
\langle 32\rangle(\theta)=i e^{M e^{\theta}+M^{*} e^{-\theta}} \cdot \frac{f_{\Delta_{2}+\Delta_{3}-\Delta_{1}}(\theta) f_{\Delta_{2}+\Delta_{3}+\Delta_{1}}(\theta)}{f_{2 \Delta_{2}}(\theta) f_{2 \Delta_{3}}(\theta)}
$$

where the first term is a zero-mode part depending on two constants $M$ and $M^{*}$. These constants can be found from the leading WKB asymptotics of $\langle 32\rangle(\theta)$ which can be found independently. We will discuss this part in section 8 and appendix $\mathrm{C}$.

The formula (5.61) is the key formula of this section. We will use it in the following to obtain the AdS contribution to the 3-point correlation functions.

Before we end this section, for completeness, let us discuss briefly the solution of equations (5.57) and (5.58). The right hand sides of these equations do not approach a constant when $\theta \rightarrow \pm \infty$ so we cannot directly use the convolution with the cosh kernel. Apart from (5.59) we just have to consider in addition

$$
\tilde{f}_{a}^{+} \tilde{f}_{a}^{-}=e^{\frac{a}{2} \pi \cosh \theta}
$$


with the solution

$$
\tilde{f}_{a}(\theta)=e^{-\frac{a}{2} \theta \sinh \theta}
$$

This will then solve the functional equations for $\langle 3 \overline{2}\rangle$ and $\langle 2 \overline{1}\rangle$. However in this case the zero mode part is undetermined. We will not consider this issue further since we do not need these expressions in the remaining part of the paper.

\subsection{Reconstruction formulas}

In this section we will show how one can reconstruct the string solution in the $\mathrm{AdS}_{2}$ target space from the Pohlmeyer data. The explicit expressions for the string solutions are important for two reasons. Firstly, the correlation function has to be regularized by making a cut-off at $z=\mathcal{E}$. This has to be translated into a worldsheet cut-off around each puncture. Secondly, we need to have control over the coordinates of the operator insertion points $x_{k}$ in gauge theory. In particular the standard conformal dependence on $x_{k}$ should arise automatically.

We will show that the string solution can be reconstructed from two given solutions $\Psi_{A}$ and $\Psi_{B}$ of the linear system for $\theta=0(\xi=1)$ normalized by $\left\langle\Psi_{A} \Psi_{B}\right\rangle=1$. Equivalently, it is determined by the coefficients $\alpha, \beta, \gamma$ and $\delta$ of

$$
\Psi_{A}=\alpha \Psi_{1}+\beta \Psi_{\overline{1}} \quad \Psi_{B}=\gamma \Psi_{1}+\delta \Psi_{\overline{1}}
$$

satisfying $\alpha \delta-\beta \gamma=1$. These two solutions can also be combined into a $2 \times 2$ matrix as

$$
\hat{\Psi}=\left(\Psi_{A} \Psi_{B}\right) \equiv\left(\begin{array}{ll}
a & b \\
c & d
\end{array}\right)
$$

Global embedding coordinates. We will present reconstruction formulas in the global embedding coordinates

$$
Y^{1}=\frac{-1}{2 z}\left(1-x^{2}-z^{2}\right) \quad Y^{2}=\frac{1}{2 z}\left(1+x^{2}+z^{2}\right) \quad Y^{3}=\frac{x}{z}
$$

satisfying

$$
\left(Y^{1}\right)^{2}-\left(Y^{2}\right)^{2}+\left(Y^{3}\right)^{2}=-1 \quad\left(d Y^{1}\right)^{2}-\left(d Y^{2}\right)^{2}+\left(d Y^{3}\right)^{2}=\frac{d x^{2}+d z^{2}}{z^{2}}
$$

Once we know $Y^{i}$, the Poincare coordinates may be easily extracted through

$$
Y^{2}-Y^{1}=\frac{1}{z} \quad Y^{3}=\frac{x}{z}
$$

Reconstruction formulas. The reconstruction formula for the string solution is

$$
Y^{I}=\frac{1}{2} \operatorname{tr}\left(\tilde{\sigma}^{I} C \hat{\Psi}^{T} D \hat{\Psi}\right)
$$

where

$$
C=\left(\begin{array}{cc}
0 & 1 \\
-1 & 0
\end{array}\right) \quad D=\left(\begin{array}{cc}
0 & i \\
i & 0
\end{array}\right)
$$


and $\tilde{\sigma}$ are related to the standard Pauli matrices by

$$
\tilde{\sigma}^{1}=\sigma^{1} \quad \tilde{\sigma}^{2}=i \sigma^{2} \quad \tilde{\sigma}^{3}=\sigma^{3}
$$

Using the equations

$$
\partial \hat{\Psi}+J \hat{\Psi}=0 \quad \bar{\partial} \hat{\Psi}+\bar{J} \hat{\Psi}=0
$$

written in the original gauge (5.9), we may verify that

$$
\begin{aligned}
\left(Y^{I}\right)^{2} & =-1 \\
\left(\partial Y^{I}\right)^{2} & =T(w) \\
\left(\bar{\partial} Y^{I}\right)^{2} & =\bar{T}(\bar{w}) \\
\left(\partial Y^{I} \bar{\partial} Y^{I}\right) & =\frac{1}{2}\left(e^{2 \gamma}+T \bar{T} e^{-2 \gamma}\right) \\
\partial \bar{\partial} Y^{I} & =\left(\partial Y^{K} \bar{\partial} Y^{K}\right) Y^{I}
\end{aligned}
$$

From the formula (5.69) we may now express the $\mathrm{AdS}_{2}$ coordinates directly in terms of the components of $\hat{\Psi}$ given above:

$$
\frac{1}{z} \equiv Y^{2}-Y^{1}=2 i a c \quad \frac{x}{z} \equiv Y^{3}=i(a d+b c)
$$

Note that these expressions are invariant under the gauge transformation

$$
\Psi \rightarrow\left(\begin{array}{cc}
\lambda & 0 \\
0 & \lambda^{-1}
\end{array}\right) \Psi
$$

thus we can use them also in our final gauge (5.17).

The operator insertion points $\boldsymbol{x}_{\boldsymbol{k}}$ and the target space cut-off $\mathcal{E}$. We may now use the above formulas to express the gauge theory operator insertion points $x_{k}$ in terms of the two solutions of the linear system $\Psi_{A}, \Psi_{B}$ which determine the classical string embedding. Fortunately, close to the puncture we have explicit formulas (5.18)-(5.19) for the basis of solutions around each puncture. Using these formulas we see that for $\xi=1$, the dominant solution around the puncture $w_{k}$ is $\bar{k}$. So only the $\beta$ and $\delta$ coefficients of $\Psi_{A, B}$ in (5.64) will be relevant.

Using the formulas (5.78) and the explicit expression (5.19) we get the link between target space $z$ coordinate and the worldsheet coordinate around the puncture $w=w_{k}$

$$
z=\frac{1}{i \beta_{k}^{2}}\left|w-w_{k}\right|^{\Delta_{k}} \quad \text { where } \quad \beta_{k}=\left\langle k \Psi_{A}\right\rangle
$$

This allows us to relate the target space cut-off $z=\mathcal{E}$ to the worldsheet cut-offs $\varepsilon_{k}$ :

$$
\Delta_{k} \log \varepsilon_{k}=\log \mathcal{E}+\log \left|\left\langle k \Psi_{A}\right\rangle\right|^{2}
$$

Similarly, we obtain expressions for the coordinates of the gauge theory operator insertion points

$$
x_{k}=\frac{\left\langle k \Psi_{B}\right\rangle}{\left\langle k \Psi_{A}\right\rangle}
$$

The two above expressions (5.81) and (5.82) are the key results of the present section which will be essential for the determination of the 'divergent' part of the AdS action integral in section 8 (recall also the overview in section 4 above). 


\section{The AdS action}

After the above preparations we are now ready to tackle the calculation of the AdS contribution to the 3-point correlation function using Pohlmeyer reduction.

We have to compute the action of the $\mathrm{AdS}_{2}$ part of the solution over the worldsheet, which is a 'regularized' 3 -punctured sphere with 3 disks cut out around the punctures ${ }^{6}$ $\left|w-w_{i}\right|>\varepsilon_{i}$. The worldsheet cut-off's around each puncture are not independent but are determined by the single target-space cut-off $z=\mathcal{E}$

$$
\frac{\sqrt{\lambda}}{\pi} \int_{\Sigma \backslash\left\{\varepsilon_{i}\right\}} \frac{\partial x \bar{\partial} x+\partial z \bar{\partial} z}{z^{2}}
$$

Let us emphasize that this is not the area of the worldsheet as there is a nonzero energymomentum tensor. Using the elements of the Pohlmeyer flat connection the above integral can be written as

$$
\frac{\sqrt{\lambda}}{\pi} \int_{\Sigma \backslash\left\{\varepsilon_{i}\right\}} 2 \operatorname{tr} \Phi_{w} \Phi_{\bar{w}}
$$

Since in the above expression we have both an unknown integrand (i.e. which depends on the solution of the modified sinh-Gordon equation which we do not know explicitly) and an unknown integration domain (since the worldsheet cut-offs depend on the targetspace solution), it is convenient, as outlined in section 4, to split the integral into a cutoff independent finite piece which involves the unknown integrand but can be integrated over the whole punctured sphere and a cut-off dependent part with an explicitly known integrand.

$$
\frac{\sqrt{\lambda}}{\pi} \int_{\Sigma}\left(2 \operatorname{tr} \Phi_{w} \Phi_{\bar{w}}-\sqrt{T \bar{T}} d^{2} w\right)+\frac{\sqrt{\lambda}}{\pi} \int_{\Sigma \backslash\left\{\varepsilon_{i}\right\}} \sqrt{T \bar{T}} d^{2} w
$$

As outlined in section 4, we also adopt a similar regularization for the $S^{5}$ part thus the cut-off dependent part will appear in the final answer with coefficient 2 . We will evaluate the first integral in section 7 , and the second integral in section 8 .

\section{The regularized Pohlmeyer contribution}

In order to evaluate the first integral in (6.3), we will proceed as for Polygonal Wilson loops, and pass to a gauge ${ }^{7}$ where the $\Phi_{w}$ part of the flat connection is diagonal

$$
\Phi_{w} \rightarrow W \Phi_{w} W^{-1}
$$

Fortunately it turns out that the diagonalized $\Phi_{w}$ does not depend on the unknown Pohlmeyer function $\gamma$ and is expressed as

$$
\Phi_{w}=\left(\begin{array}{cc}
-\frac{\sqrt{T}}{2} & 0 \\
0 & \frac{\sqrt{T}}{2}
\end{array}\right)
$$

\footnotetext{
${ }^{6}$ For the puncture at $w=\infty$ we define the worldsheet cut-off through $|w|<1 / \varepsilon_{\infty}$.

${ }^{7}$ By this we mean redefining the solution of the linear system $\tilde{\Psi}=W \Psi$ with some given matrix $W$ depending on the worldsheet coordinates $w, \bar{w}$.
} 
The diagonal components of $\Phi_{\bar{w}}$ become more complicated

$$
\Phi_{\bar{w}}=\left(\begin{array}{ll}
-\frac{\left(e^{2 \gamma}+T \bar{T} e^{-2 \gamma}\right)}{4 \sqrt{T}} & \frac{\left(e^{2 \gamma}-T \bar{T} e^{-2 \gamma}\right)}{4 \sqrt{T}} \\
-\frac{\left(e^{2 \gamma}-T \bar{T} e^{-2 \gamma}\right)}{4 \sqrt{T}} & \frac{\left(e^{2 \gamma}+T \bar{T} e^{-2 \gamma}\right)}{4 \sqrt{T}}
\end{array}\right)=\left(\begin{array}{ll}
-\frac{1}{2} \sqrt{\bar{T}} \cosh \tilde{\gamma} & \frac{1}{2} \sqrt{\bar{T}} \sinh \tilde{\gamma} \\
-\frac{1}{2} \sqrt{\bar{T}} \sinh \tilde{\gamma} & \frac{1}{2} \sqrt{\bar{T}} \cosh \tilde{\gamma}
\end{array}\right)
$$

however, the important observation made in [51] is that the diagonal components of each expression can be treated as a single function defined on a double cover $\widetilde{\Sigma}$

$$
y^{2}=T(w)
$$

of the worldsheet $\Sigma$.

In this manner one can rewrite the integral

$$
\int_{\Sigma}\left(2 \operatorname{tr} \Phi_{w} \Phi_{\bar{w}}-\sqrt{T \bar{T}} d^{2} w\right)
$$

as an integral over $\widetilde{\Sigma}$ of a wedge product of two closed 1-forms:

$$
\int_{\Sigma}\left(2 \operatorname{tr} \Phi_{w} \Phi_{\bar{w}}-\sqrt{T \bar{T}} d^{2} w\right)=\frac{i}{2} \cdot \int_{\widetilde{\Sigma}} \omega \wedge \eta
$$

with

$$
\omega=\sqrt{T(w)} d w \quad \eta=\frac{1}{2} \sqrt{\bar{T}(\bar{w})}(\cosh \tilde{\gamma}-1) d \bar{w}+\frac{1}{4} \frac{1}{\sqrt{T(w)}}(\partial \tilde{\gamma})^{2} d w
$$

where for simplicity we used the original Pohlmeyer function (see (5.5)). The $d w$ component of $\eta$ does not influence the integral but is chosen so that $\eta$ is also closed $(d \eta=0)$.

If $\widetilde{\Sigma}$ had genus $g$ (which is the generic case for Polygonal Wilson loops), one would use Riemann bilinear identity (or reciprocity) to reduce the integral to products of integrals over cycles

$$
\int_{\Sigma_{g}} \omega \wedge \eta=\sum_{i=1}^{g} \int_{A_{i}} \omega \int_{B_{i}} \eta-\int_{A_{i}} \eta \int_{B_{i}} \omega
$$

However in our case $\widetilde{\Sigma}$ has genus 0 , and the 1 -forms may have singularities at 8 points (two copies of the 3 punctures and 2 branch points of the covering $y^{2}=T(w)$ ). One possibility to proceed is to prove an analog of Riemann reciprocity directly for this case. The resulting expressions are, however, quite messy. In the end, we decided to adopt a slightly different strategy by treating the punctures as infinitesimal branch cuts and using Riemann reciprocity for a genus 3 Riemann surface (see figure 1) with an additional treatment of the singularities at the zeroes of $T(w)$. Let us note that the $\eta 1$-form is neither holomorphic or antiholomorphic and generic textbook formulas are not directly applicable.

The idea of the derivation of the Riemann reciprocity formula is to rewrite one of the forms as an exact form:

$$
\omega=d F
$$




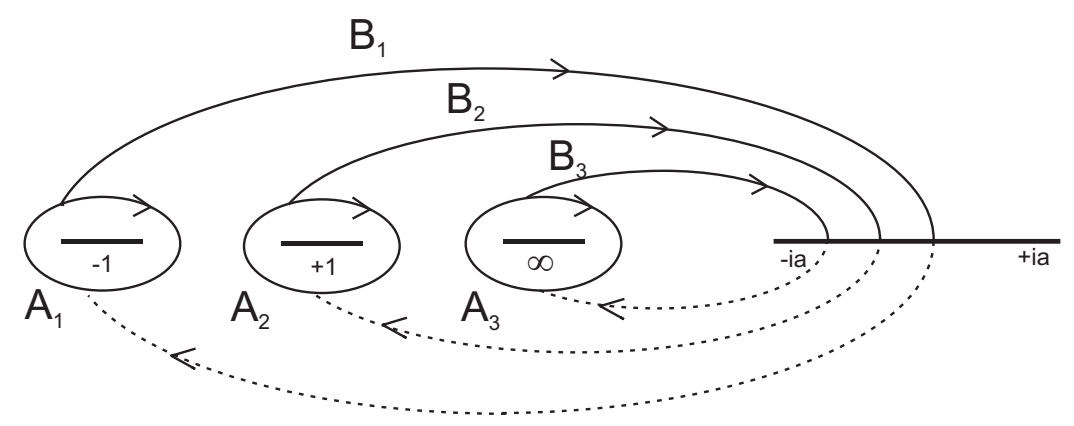

Figure 1. Cycles on a genus 3 surface. For concrete computations it is convenient to make all the $B_{i}$ cycles to pass through the same point on the last cut e.g. $w=0$.

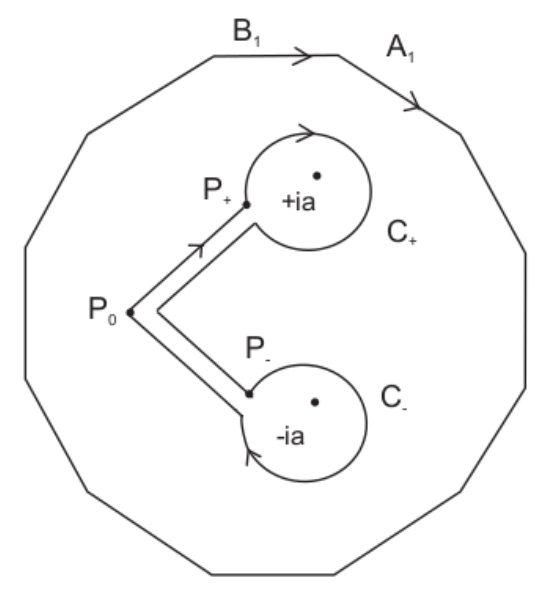

Figure 2. The polygon is the standard representation of a genus 3 surface whose boundary is the curve $L_{g=3}$ composed of the cycles $A_{i}$ and $B_{i}$ each traversed twice. The infinitesimal circles $C_{ \pm}$surround the singularities of $\eta$. $P_{0}$ is the (arbitrary but fixed) base point for constructing the function $F$ such that $\omega=d F$.

where $F(P)=\int_{P_{0}}^{P} \omega$. This can always be done on the Riemann surface minus some contour. Then one transforms the surface integral into a 1-dimensional integral over that contour using Stokes theorem:

$$
\int_{\widetilde{\Sigma}} \omega \wedge \eta=\int_{\widetilde{\Sigma} \backslash L} \omega \wedge \eta=\int_{\widetilde{\Sigma} \backslash L} d(F \eta)=\int_{L} F \eta
$$

In our case, since $\omega$ is regular ${ }^{8}$ at the zeroes of $T(w)$, the contour $L$ may be taken to be the sum of the standard contour for a genus 3 surface $L_{g=3}$ and two infinitesimal circles $C_{ \pm}$around the zeros of $T(w)$ at $w= \pm i a$ as shown on figure 2.

\footnotetext{
${ }^{8}$ Recall that we are on $\widetilde{\Sigma}$.
} 
Therefore

$$
\int_{\widetilde{\Sigma}} \omega \wedge \eta=\int_{L_{g=3}} F \eta+\int_{C_{+}} F \eta+\int_{C_{-}} F \eta
$$

The first integral gives directly the standard bilinear expression

$$
\int_{L_{g=3}} F \eta=\sum_{i=1}^{3} \int_{A_{i}} \omega \int_{B_{i}} \eta-\int_{A_{i}} \eta \int_{B_{i}} \omega
$$

where we used the fact that

$$
\int_{C_{+}} \omega=\int_{C_{-}} \omega=0
$$

Let us now concentrate on the remaining two terms. Now,

$$
\begin{aligned}
\int_{C_{+}} F \eta & =\int_{C_{+}}\left(\int_{P_{0}}^{P} \omega\right) \eta=\int_{C_{+}}\left[\left(\int_{P_{0}}^{P_{+}}+\int_{P_{+}}^{P}\right) \omega\right] \eta \\
& =\int_{P_{0}}^{P_{+}} \omega \int_{C_{+}} \eta+\int_{C_{+}}\left(\int_{P_{+}}^{P} \omega\right) \eta=\int_{P_{0}}^{P_{+}} \omega \int_{C_{+}} \eta-i \pi \frac{1}{6}
\end{aligned}
$$

where the last integral is computed in appendix B.1. Then, adding a similar expression for the second zero we get

$$
\begin{aligned}
\left(\int_{C_{+}}+\int_{C_{-}}\right) F \eta & =\int_{P_{0}}^{P_{+}} \omega \int_{C_{+}} \eta+\int_{P_{0}}^{P_{-}} \omega \int_{C_{-}} \eta-2 \cdot i \pi \frac{1}{6} \\
& =\int_{P_{-}}^{P_{+}} \omega \int_{C_{+}} \eta-2 \cdot i \pi \frac{1}{6} \\
& =-2 \cdot i \pi \frac{1}{6}
\end{aligned}
$$

where we used the fact that the 1-form $\eta$ is regular everywhere apart from the zeros of $T(w)$ and so

$$
\int_{C_{+}} \eta+\int_{C_{-}} \eta=0
$$

Moreover, one can even show that $\int_{C_{+}} \eta=0$ (see appendix B.2). In this way we arrived at the final equality.

Further, inserting this result into the integral (7.11), and computing the periods in (7.12) using again the regularity of $\eta$ outside the zeros of $T(w)$ and explicitly computing the integrals of the 1 -form $\omega$

$$
\int_{A_{1}} \omega=\int_{A_{2}} \omega=-2 \pi i \frac{\Delta}{2}, \quad \int_{A_{3}} \omega=2 \pi i \frac{\Delta_{\infty}}{2}
$$

we find that

$$
\begin{aligned}
\int_{\widetilde{\Sigma}} \omega \wedge \eta & =\sum_{i=1}^{3} \int_{A_{i}} \omega \int_{B_{i}} \eta-2 \cdot i \pi \frac{1}{6} \\
& =2 \pi i\left[-\frac{\Delta}{2}\left(\int_{B_{1}} \eta+\int_{B_{2}} \eta\right)+\frac{\Delta_{\infty}}{2} \int_{B_{3}} \eta-\frac{1}{6}\right]
\end{aligned}
$$


The integrals over the cycles $B_{i}$ may be expressed by integrals between the punctures. From figure 1 and the antisymmetry of $\eta$ under changing of the Riemann sheet we find

$$
\begin{aligned}
2 \int_{C_{-11}} \eta & =\int_{B_{1}} \eta+\int_{B_{2}} \eta, \quad \int_{B_{1}} \eta=\int_{B_{2}} \eta, \\
2 \int_{C_{1 \infty}} \eta & =\int_{B_{1}} \eta-\int_{B_{3}} \eta
\end{aligned}
$$

Hence,

$$
\int_{\widetilde{\Sigma}} \omega \wedge \eta=-2 i\left[\frac{\pi}{6}-\frac{\pi}{2}\left(\left(\Delta_{\infty}-2 \Delta\right) \int_{C_{-11}} \eta-2 \Delta_{\infty} \int_{C_{1 \infty}} \eta\right)\right]
$$

Therefore the regularized Pohlmeyer contribution becomes

$$
\int_{\Sigma}\left(2 \operatorname{tr} \Phi_{w} \Phi_{\bar{w}}-\sqrt{T(w) \bar{T}(\bar{w})} d^{2} w\right)=\frac{\pi}{6}-\frac{\pi}{2}\left(\left(\Delta_{\infty}-2 \Delta\right) \int_{C_{-11}} \eta-2 \Delta_{\infty} \int_{C_{1 \infty}} \eta\right)
$$

At this stage we have reduced the computation of the regularized Pohlmeyer contribution to the evaluation of the integrals of the 1-form $\eta$ between the punctures. This cannot be done directly, as we do not know of course the explicit form of the Pohlmeyer solution $\gamma$ or $\tilde{\gamma}$. However, as shown in [51], the integrals of $\eta$ can be related to the $\theta \rightarrow-\infty(\xi \rightarrow 0)$ asymptotics of the parallel transport of a solution along the curve which is a WKB line [53].

The main idea is to apply the well-know semiclassical methods where the role of the Plank constant is played by the spectral parameter $\xi$. Then, the linear problem

$$
(d+J) \Psi=0
$$

can be approximately solved with the leading contribution coming from the $\Phi_{w}$ part

$$
\Psi \sim e^{\mp \frac{1}{2 \xi} \int \sqrt{T(w)} d w}
$$

Clearly, the approximation is the best once we are on the WKB line defined as

$$
\operatorname{Im}\left(\frac{1}{\xi} \sqrt{T(w)} \dot{w}\right)=0
$$

For our purposes, however, it is crucial to know also the subleading term related to the $\Phi_{\bar{w}}$ part of the flat connection

$$
e^{ \pm \xi \int \tilde{\eta}}=e^{ \pm \xi \int\left(\tilde{\eta}-\frac{1}{2} \sqrt{\bar{T}(\bar{w})} d \bar{w}\right)} e^{ \pm \frac{\xi}{2} \int \sqrt{\bar{T}(\bar{w})} d \bar{w}}=e^{ \pm \xi \int \eta} e^{ \pm \frac{\xi}{2} \int \sqrt{\bar{T}(\bar{w})} d \bar{w}}
$$

where $\tilde{\eta}$ is the 1 -form $\eta$ without the subtraction term $\sqrt{\bar{T}(\bar{w})} / 2$, exactly as it shows up in $\Phi_{\bar{w}}$.

The basic object we want to compute in this limit is the skew product between two solutions at the punctures $j, k$. Then, the prescription is the following:

(i) take the known solution $\Psi_{j}\left(w_{j}^{\prime}\right)$ at $w=w_{j}^{\prime}$ in the vicinity of the puncture $w_{j}$

(ii) transport this solution via the parallel transport equation along a curve given by the WBK line equation to a point $w=w_{k}^{\prime}$ near the puncture at $w_{k}$ taking into account the leading as well as the subleading terms 
(iii) compare the resulting with the known solution $\Psi_{k}\left(w_{k}^{\prime}\right)$ at $w=w_{k}^{\prime}$.

The resulting formula reads

$$
\begin{aligned}
\lim _{\xi \rightarrow 0}\langle j k\rangle= & e^{\frac{1}{\xi}\left[\frac{1}{2} \int_{w_{j}^{\prime}}^{w_{k}^{\prime}} \sqrt{T(w)} d w+\frac{\Delta_{j}}{4} \log \left(w_{j}-w_{j}^{\prime}\right)+\frac{\Delta_{k}}{4} \log \left(w_{k}-w_{k}^{\prime}\right)\right]} \cdot \\
& e^{\xi\left[\frac{1}{2} \int_{w_{j}^{\prime}}^{w_{k}^{\prime}} \sqrt{\bar{T}(\bar{w})} d \bar{w}+\frac{\Delta_{j}}{4} \log \left(\bar{w}_{j}-\bar{w}_{j}^{\prime}\right)+\frac{\Delta_{k}}{4} \log \left(\bar{w}_{k}-\bar{w}_{k}^{\prime}\right)\right]} \cdot e^{\xi \int_{w_{j}}^{w_{k}} \eta}
\end{aligned}
$$

where the logarithmic terms are due to the exactly known form of the solution near the punctures. Moreover, these subtractions render the expression finite and therefore allow to extend the integration exactly to the punctures. This formula may be now compared with the exact expression for the skew product at any $\xi$ (5.61), which contains two undetermined zero mode constants $M, M^{*}$. Fortunately, they are given by the first two terms of the WKB approximation. Then, the path integral of $\eta$ may be given by a combination of the $\theta \rightarrow-\infty$ asymptotic of $f_{a}(\theta)$ function.

In this way we obtain the following explicit expressions for the period integrals

$$
\begin{aligned}
& \int_{C_{-11}} \eta=h\left(2 \Delta-\Delta_{\infty}\right)+h\left(2 \Delta+\Delta_{\infty}\right)-2 h(2 \Delta) \\
& \int_{C_{1 \infty}} \eta=h\left(\Delta_{\infty}\right)+h\left(2 \Delta+\Delta_{\infty}\right)-h(2 \Delta)-h\left(2 \Delta_{\infty}\right)
\end{aligned}
$$

where

$$
h(a)=\int_{-\infty}^{\infty} \frac{d \theta}{\pi} \cosh \theta \log \left(1-e^{-a \pi \cosh \theta}\right)
$$

Together with (7.23) it gives our final explicit expression for the regularized Pohlmeyer contribution.

\subsection{Comparison with numerics}

Since the above derivation of (7.23)-(7.30) was quite complicated and involved many new ingredients, we decided to test the result by numerically solving the modified sinh-Gordon equation on the 3-punctured sphere and directly computing the regularized Pohlmeyer integral from the numerical solution. We give some details on the numerical setup in appendix A, while here we just summarize the results and the comparison with the analytical predictions (7.23)-(7.30).

In figure 3 we show a plot of the integrand entering the regularized Pohlmeyer action

$$
\int_{\Sigma}\left\{\frac{1}{2}\left(e^{2 \gamma(w, \bar{w})}+T(w) \bar{T}(\bar{w}) e^{-2 \gamma(w, \bar{w})}\right)-\sqrt{T(w) \bar{T}(\bar{w})}\right\} d^{2} w
$$

in a qudrant of the (compactified) complex plane. The upper and right borders are mapped to the puncture at $w=\infty$, while the puncture at $w=+1$ is right in the middle of the lower border. The solution in the remaining three quadrants follows by symmetry. In the table we have shown a comparison of the numerical evaluation of (7.32) together with the analytical results following from (7.23)-(7.30). The numerics becomes more difficult and 


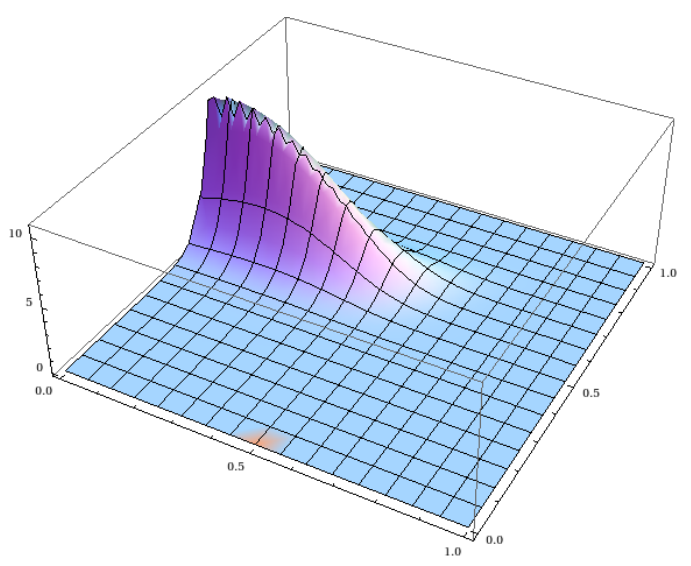

\begin{tabular}{|c|c|c|c|}
\hline$\Delta$ & $\Delta_{\infty}$ & numerics & our formula \\
\hline 0.2 & 0.3 & 0.04536 & 0.0450779 \\
0.5 & 0.9 & 0.107649 & 0.107622 \\
1. & 1. & 0.426311 & 0.426166 \\
1. & 1.05 & 0.429572 & 0.429503 \\
2. & 2. & 0.517689 & 0.517688 \\
2. & 3. & 0.488985 & 0.488985 \\
4. & 4. & 0.523584 & 0.523584 \\
4. & 7.99 & 0.0152435 & 0.0152435 \\
\hline
\end{tabular}

Figure 3. Regularized action density for $\Delta=\Delta_{\infty}=4.0$ and a comparison between the numerically evaluated regularized action and the analytical results of the formulas (7.23)-(7.30).

less reliable for small $\Delta$ 's. In particular the deviations in the first rows of the table are within numerical errors estimated by changing the number of points of the numerical grid. The remaining results show excellent agreement with the analytical formulas following from periods and solutions of the functional equations for the overlaps.

\section{The regularized divergent contribution}

In this section we will deal with the remaining contribution to the action - an integral of $\sqrt{T \bar{T}}$ on the punctured sphere with specific cut-offs around each puncture $\left|w-w_{i}\right|>\varepsilon_{i}$. Since we adopt a similar regularization for the $S^{5}$ contribution (see (4.4)), we have in fact two such contributions

$$
\exp \left\{-\frac{2 \sqrt{\lambda}}{\pi} \int_{\Sigma \backslash\left\{\varepsilon_{i}\right\}} \sqrt{T(w) \bar{T}(\bar{w})} d^{2} w\right\}
$$

The above integral can be evaluated explicitly in the small $\varepsilon_{i}$ limit relevant for us. To do it, we note that it can be expressed as an integral of the wedge product of two closed 1-forms:

$$
\int_{\Sigma \backslash\left\{\varepsilon_{i}\right\}} \sqrt{T(w) \bar{T}(\bar{w})} d^{2} w=\text { const } \cdot \int \sqrt{T} d w \wedge \sqrt{\bar{T}} d \bar{w}
$$

and transformed into a product of residues of $\sqrt{T}$ times appropriate (regularized) integrals over intervals between punctures. The outcome is

$$
\int_{\Sigma \backslash\left\{\varepsilon_{i}\right\}} \sqrt{T(w) \bar{T}(\bar{w})} d^{2} w=\text { Finite }-\frac{\pi}{2} \Delta_{\infty}^{2} \log \varepsilon_{\infty}-\frac{\pi}{2} \Delta^{2} \log \varepsilon-\frac{\pi}{2} \Delta^{2} \log \varepsilon
$$

where

$$
\begin{array}{r}
\text { Finite }=\frac{\pi}{4} \Delta_{\infty}^{2}\left(2 \log 2+\left(1+a^{2}\right) \log 2+\left(-2-a^{2}+2 \sqrt{1+a^{2}}\right) \log a^{2}+\right. \\
\left.+\left(1+a^{2}\right) \log \left(1+a^{2}\right)-4 \sqrt{1+a^{2}} \log \left(1+\sqrt{1+a^{2}}\right)\right)
\end{array}
$$


Let us first concentrate on the logarithmically divergent part. Taking into account the coupling-constant dependent prefactor, and generalizing slightly to three generic anomalous dimensions, we get

$$
\exp \left\{\sqrt{\lambda}\left(\Delta_{1}^{2} \log \varepsilon_{1}+\Delta_{2}^{2} \log \varepsilon_{2}+\Delta_{3}^{2} \log \varepsilon_{3}\right)\right\}
$$

We may use the relations (5.81) to express the worldsheet cut-off in terms of the physical target-space cut-off $z=\mathcal{E}$ and the products between the elementary solutions $1,2,3$ and one $\left(\Psi_{A}\right)$ of the two solutions appearing in the reconstruction formulas of section 5 . We get

$$
\exp \left\{\sum \boldsymbol{\Delta}_{i} \log \mathcal{E}+\boldsymbol{\Delta}_{1} \log |\langle 1 A\rangle|^{2}+\boldsymbol{\Delta}_{2} \log |\langle 2 A\rangle|^{2}+\boldsymbol{\Delta}_{3} \log |\langle 3 A\rangle|^{2}\right\}
$$

where $\boldsymbol{\Delta}_{i} \equiv \sqrt{\lambda} \Delta_{i}$ is the unrescaled anomalous dimension.

We will now express the scalar products $\langle k A\rangle$ in terms of the gauge theory operator insertion points $x_{k}$ and products between the elementary solutions. The two solutions of the linear system $\Psi_{A}$ and $\Psi_{B}$ which determine the target-space string embedding are completely specified by their coordinates in e.g. the $1, \overline{1}$ basis:

$$
\Psi_{A}=\alpha 1+\beta \overline{1} \quad \Psi_{B}=\gamma 1+\delta \overline{1}
$$

where $\alpha \delta-\beta \gamma=1$. Similarly we can express the 2 and 3 elementary solutions entering (8.6) in terms of 1 and $\overline{1}$ :

$$
2=k 1+l \overline{1} \quad 3=m 1+n \overline{1}
$$

where $k, l, m$ and $n$ are appropriate overlaps evaluated at $\theta=0(\xi=1)$. In terms of the above quantities, the part of (8.6) depending on the products becomes

$$
\sum_{k} \boldsymbol{\Delta}_{k} \log |\langle k A\rangle|^{2}=\boldsymbol{\Delta}_{1} \log \beta^{2}+\boldsymbol{\Delta}_{2} \log (k \beta-l \alpha)^{2}+\boldsymbol{\Delta}_{3} \log (m \beta-n \alpha)^{2}
$$

Now we may use formula (5.82) to relate the hitherto unknown coefficients $\alpha, \beta$ and $\gamma$ to the operator insertion points:

$$
\begin{aligned}
& x_{1}=\frac{\left\langle 1 \Psi_{B}\right\rangle}{\left\langle 1 \Psi_{A}\right\rangle}=\frac{\delta}{\beta} \\
& x_{2}=\frac{\left\langle 2 \Psi_{B}\right\rangle}{\left\langle 2 \Psi_{A}\right\rangle}=\frac{k \delta-l \gamma}{k \beta-l \alpha} \\
& x_{3}=\frac{\left\langle 3 \Psi_{B}\right\rangle}{\left\langle 3 \Psi_{A}\right\rangle}=\frac{m \delta-n \gamma}{m \beta-n \alpha}
\end{aligned}
$$

Solving these equations with the constraint $\alpha \delta-\beta \gamma=1$ yields

$$
\begin{aligned}
\beta^{2} & =\frac{l n}{l m-k n} \cdot \frac{x_{23}}{x_{12} x_{13}} \\
(k \beta-l \alpha)^{2} & =\frac{l}{n}(l m-k n) \cdot \frac{x_{13}}{x_{12} x_{23}} \\
(m \beta-n \alpha)^{2} & =\frac{n}{l}(l m-k n) \cdot \frac{x_{12}}{x_{13} x_{23}}
\end{aligned}
$$


In the above formula $l=\langle 12\rangle_{0} \equiv\langle 12\rangle_{\theta=0}, n=\langle 13\rangle_{0}$, while

$$
l m-k n=\langle 12\rangle_{0}\langle 3 \overline{1}\rangle_{0}-\langle 2 \overline{1}\rangle_{0}\langle 13\rangle_{0}=\langle 32\rangle_{0}
$$

using Schouten's identity.

Plugging the above expressions into (8.6), we obtain finally the standard CFT spacetime dependence of the 3 -point function

$$
\frac{1}{\left(\frac{x_{12}}{\mathcal{E}}\right)^{\boldsymbol{\Delta}_{1}+\boldsymbol{\Delta}_{2}-\boldsymbol{\Delta}_{3}}\left(\frac{x_{13}}{\mathcal{E}}\right)^{\boldsymbol{\Delta}_{1}+\boldsymbol{\Delta}_{3}-\boldsymbol{\Delta}_{2}}\left(\frac{x_{23}}{\mathcal{E}}\right)^{\boldsymbol{\Delta}_{2}+\boldsymbol{\Delta}_{3}-\boldsymbol{\Delta}_{1}}} \cdot \ldots
$$

multiplied by an additional contribution coming from the products of the elementary solutions

$$
\exp ^{\sqrt{\lambda}\left(\left(\Delta_{1}+\Delta_{2}-\Delta_{3}\right) \log \langle 12\rangle_{0}+\left(\Delta_{1}+\Delta_{3}-\Delta_{2}\right) \log \langle 13\rangle_{0}+\left(\Delta_{2}+\Delta_{3}-\Delta_{1}\right) \log \langle 32\rangle_{0}\right)}
$$

Going back to our solution of the functional equations (and returning to the symmetric case of $\Delta_{1}=\Delta_{2}=\Delta$ and $\left.\Delta_{3}=\Delta_{\infty}\right)$ we see that the products have the following structure at $\theta=0$ :

$$
\begin{aligned}
\langle 12\rangle_{0} & =e^{M_{-11}+M_{-11}^{*}} \cdot e^{K_{-11}} \\
\langle 13\rangle_{0}=\langle 23\rangle_{0} & =e^{M_{1 \infty}+M_{1 \infty}^{*} \cdot e^{K_{1 \infty}}}
\end{aligned}
$$

where

$$
\begin{aligned}
K_{-11} & =k\left(2 \Delta-\Delta_{\infty}\right)+k\left(2 \Delta+\Delta_{\infty}\right)-2 k(2 \Delta) \\
K_{1 \infty} & =k\left(\Delta_{\infty}\right)+k\left(2 \Delta+\Delta_{\infty}\right)-k(2 \Delta)-k\left(2 \Delta_{\infty}\right)
\end{aligned}
$$

with

$$
k(a)=\int_{-\infty}^{\infty} \frac{d \theta}{2 \pi} \frac{\log \left(1-e^{-a \pi \cosh \theta}\right)}{\cosh \theta}
$$

The zero-mode constants $M_{-11}$ and $M_{1 \infty}$ are evaluated in appendix $\mathrm{C}$ and after substituting into (8.18), it turns out that they exactly cancel the finite term (8.4). Thus the remaining contribution to the OPE coefficient becomes finally

$$
\exp \left\{\sqrt{\lambda}\left[\left(2 \Delta-\Delta_{\infty}\right) K_{-11}+2 \Delta_{\infty} K_{1 \infty}\right]\right\}
$$

\section{The final AdS contribution to the OPE coefficients}

We may now sum together the two contributions to the OPE coefficients - (7.23)-(7.30) coming from the regularized Pohlmeyer integral and (8.24) coming from the regularized divergent integral. Both contributions have the same structure yielding

$$
C_{\mathrm{AdS}}^{\mathrm{OPE}}=\exp \left\{-\frac{\sqrt{\lambda}}{6}-\sqrt{\lambda}\left[\left(2 \Delta-\Delta_{\infty}\right) \tilde{P}_{-11}+2 \Delta_{\infty} \tilde{P}_{1 \infty}\right]\right\}
$$

with

$$
\begin{aligned}
\tilde{P}_{-11} & =\tilde{h}\left(2 \Delta-\Delta_{\infty}\right)+\tilde{h}\left(2 \Delta+\Delta_{\infty}\right)-2 \tilde{h}(2 \Delta) \\
\tilde{P}_{1 \infty} & =\tilde{h}\left(\Delta_{\infty}\right)+\tilde{h}\left(2 \Delta+\Delta_{\infty}\right)-\tilde{h}(2 \Delta)-\tilde{h}\left(2 \Delta_{\infty}\right)
\end{aligned}
$$


where

$$
\tilde{h}(a)=\frac{1}{2} h(a)-k(a)=\frac{1}{2 \pi} \int_{-\infty}^{\infty} \frac{\sinh ^{2} \theta}{\cosh \theta} \log \left(1-e^{-a \pi \cosh \theta}\right) d \theta
$$

Several comments are in order here. Firstly, the above expression does not depend on any details of the operators entering the OPE coefficient apart from their anomalous dimensions, thus it is universal for this class of operators. Secondly, the above expression has to be supplanted by the regularized $S^{5}$ contribution

$$
C_{S^{5}}^{\mathrm{OPE}} \equiv e^{-\frac{\sqrt{\lambda}}{\pi} \int_{\Sigma}\left(S^{5} \text { contribution }-\sqrt{T \bar{T}}\right)}
$$

so one cannot draw conlusions on the behaviour of real OPE coefficients $C^{\mathrm{OPE}}=C_{\mathrm{AdS}}^{\mathrm{OPE}}$. $C_{S^{5}}^{\mathrm{OPE}}$, since the latter part is currently unknown. Thirdly, the factor $\exp (-\sqrt{\lambda} / 6)$ seems quite surprising, however its presence is essential for sensible extremal and small $\Delta_{i}$ limits which we will examine shortly.

In this paper we have mostly considered the symmetric case of two equal anomalous dimensions. It should not be difficult to extend these considerations to the generic case of three distinct anomalous dimensions. Repeating e.g. the analysis of the regularized Pohlmeyer contribution suggests the following structure.

Let us introduce the parameters $\alpha_{i}$ :

$$
\alpha_{1}=\Delta_{2}+\Delta_{3}-\Delta_{1} \quad \alpha_{2}=\Delta_{1}+\Delta_{3}-\Delta_{2} \quad \alpha_{3}=\Delta_{1}+\Delta_{2}-\Delta_{3}
$$

Then the general answer should be

$$
C_{\mathrm{AdS}}^{\mathrm{OPE}}=\exp \left\{-\sqrt{\lambda}\left(\frac{1}{6}+F\left(\alpha_{1}, \alpha_{2}, \alpha_{3}\right)\right)\right\}
$$

where

$$
\begin{aligned}
F\left(\alpha_{1}, \alpha_{2}, \alpha_{3}\right)= & \alpha_{1} \tilde{h}\left(\alpha_{1}\right)+\alpha_{2} \tilde{h}\left(\alpha_{2}\right)+\alpha_{3} \tilde{h}\left(\alpha_{3}\right)+\left(\alpha_{1}+\alpha_{2}+\alpha_{3}\right) \tilde{h}\left(\alpha_{1}+\alpha_{2}+\alpha_{3}\right) \\
& -\left(\alpha_{1}+\alpha_{2}\right) \tilde{h}\left(\alpha_{1}+\alpha_{2}\right)-\left(\alpha_{1}+\alpha_{3}\right) \tilde{h}\left(\alpha_{1}+\alpha_{3}\right)-\left(\alpha_{3}+\alpha_{2}\right) \tilde{h}\left(\alpha_{3}+\alpha_{2}\right)
\end{aligned}
$$

The structure of $F\left(\alpha_{1}, \alpha_{2}, \alpha_{3}\right)$ is very similar to the structure of formula (7.11) in [43] but with a different function $\tilde{h}$ instead of a logarithm. Below we will see that (7.11) arises from our formula in the limit of small anomalous dimensions.

Extremal limit. In the extremal limit $\Delta_{\infty}=2 \Delta$, all the terms with $\tilde{h}(a)$ 's with nonzero arguments will cancel between each other leaving the term $\left(2 \Delta-\Delta_{\infty}\right) \tilde{h}\left(2 \Delta-\Delta_{\infty}\right)$. Now $\tilde{h}(a) \sim-\frac{1}{6 a}$ (see appendix D), so the remaining term will cancel with the $-\sqrt{\lambda} / 6$ giving the expected result

$$
C_{\mathrm{AdS}}^{\mathrm{OPE}}\left(\Delta, \Delta, \Delta_{\infty}=2 \Delta\right)=1
$$

Small $\boldsymbol{\Delta}_{\boldsymbol{i}}$ limit. For small arguments, $\tilde{h}(a)$ behaves like (see appendix D)

$$
\tilde{h}(a) \sim-\frac{1}{6 a}-\frac{1}{2} \log a
$$


It turns out that contributions coming from the leading term will cancel out completely. The subleading logarithmic terms yield an expression

$$
C_{\mathrm{AdS}}^{\mathrm{OPE}}\left(\Delta, \Delta, \Delta_{\infty}\right) \rightarrow\left(\frac{\left(2 \Delta-\Delta_{\infty}\right)^{2 \boldsymbol{\Delta}-\boldsymbol{\Delta}_{\infty}}\left(2 \Delta+\Delta_{\infty}\right)^{2 \Delta+\Delta_{\infty}} \Delta_{\infty}^{2 \Delta_{\infty}}}{(2 \Delta)^{4 \Delta}\left(2 \Delta_{\infty}\right)^{2 \Delta_{\infty}}}\right)^{\frac{1}{2}}
$$

which coincides with formula (7.11) in [43].

Large $\Delta_{i}$ limit and the Painleve transcendental. For large arguments $\tilde{h}(a) \propto a^{\#}$. $e^{-\pi a}$, and thus their contribution is exponentially suppressed yielding a surprisingly simple universal limit independent of the conformal dimensions of operators:

$$
C_{\mathrm{AdS}}^{\mathrm{OPE}}\left(\Delta, \Delta, \Delta_{\infty}\right) \rightarrow \exp \left(-\frac{\sqrt{\lambda}}{6}\right)
$$

The simplicity of this result suggests that there should exist a much simpler direct derivation of the above result. ${ }^{9}$ This turns out indeed to be the case.

In order to study the large $\Delta$ limit it is most convenient to study the modified sinhGordon equation in its original formulation (5.2)

$$
\partial \bar{\partial} \tilde{\gamma}=\sqrt{T \bar{T}} \sinh \tilde{\gamma}
$$

where

$$
T(w)=\frac{\Delta_{\infty}^{2}}{4} \frac{w^{2}+a^{2}}{\left(1-w^{2}\right)^{2}}
$$

The advantage of using (9.13) is that $\tilde{\gamma} \rightarrow 0$ around the punctures. Hence it would seem naively that $\tilde{\gamma}=0$ would be a possible solution of the equations of motion. This is not the case, however, as due to the expression for the string induced metric (5.1), $\tilde{\gamma}$ has to have logarithmic singularities

$$
\tilde{\gamma} \sim \pm \log |w \pm i a|
$$

at the zeros of $T(w)$. Nevertheless, in the large $\Delta$ limit, when $\sqrt{T \bar{T}}$ is generically very large, in order to minimize the string action (5.1), we expect to have an almost vanishing solution with two narrow logarithmic spikes around the two zeros of $T(w)$.

Let us concentrate on the neighbourhood of $w=i a$ and introduce a new coordinate through $w=u+i a$. Then (9.13) takes the form

$$
\partial \bar{\partial} \tilde{\gamma}=\underbrace{\frac{\Delta_{\infty}^{2}}{4} \frac{2 a}{\left(1+a^{2}\right)^{2}}}_{C^{2}} \cdot \sqrt{u} \sqrt{\bar{u}} \sinh \tilde{\gamma}
$$

Redefining coordinates again

$$
v=\frac{2}{3} C u^{\frac{3}{2}}
$$

yields the standard sinh-Gordon equation

$$
\partial \bar{\partial} \tilde{\gamma}=\sinh \tilde{\gamma}
$$

\footnotetext{
${ }^{9}$ Thanks to Pedro Vieira for asking this interesting question.
} 
In the large $\Delta$ limit the problem becomes rotationally invariant and after introducing a new variable $\tilde{\gamma}=2 U$ and $R=2 r \equiv 2|v|$ we obtain the equation for a Painlevé III transcendent normalized as in [54]:

$$
U^{\prime \prime}+\frac{1}{R} U^{\prime}=\frac{1}{2} \sinh 2 U
$$

The coefficient of the logarithmic singularity (9.15) becomes

$$
U \sim \pm \frac{1}{3} \log R
$$

Now we may use the results of [54] to evaluate directly the regularized Pohlmeyer action. We may rewrite the contribution around $w=i a$ as

$$
\int \sqrt{T \bar{T}}(\cosh \tilde{\gamma}-1) d^{2} w=\int_{0}^{\infty}(\cosh 2 U-1) \frac{3 \pi R d R}{4}
$$

Note the $3 \pi$ which comes from an angular integral corresponding to a $2 \pi$ angle in the original $u$ and $w$ coordinates. We then use the substitution from [54]

$$
\frac{1}{2} \cosh 2 U=-\frac{1}{R} \frac{d}{d R} R F_{c}(R)
$$

to get

$$
-\frac{3 \pi}{2} \int_{0}^{\infty}\left(\frac{d}{d R} R F_{c}(R)+\frac{R}{2}\right) d R
$$

This integral can be evaluated exactly using the asymptotic properties of $F_{c}(R)$ established in [54]. At large $R, F_{c}(R) \sim-R / 4$ up to exponentially small terms, while for small $R$, $R F_{c}(R) \rightarrow 1 / 18$. Thus the above integral evaluates to $\pi / 12$. Now taking into account two such contributions and the prefactor of the integral (9.21), we arrive directly at our universal large $\Delta$ limit:

$$
e^{-\frac{\sqrt{\lambda}}{\pi}\left(\frac{\pi}{12}+\frac{\pi}{12}\right)}=e^{-\frac{\sqrt{\lambda}}{6}}
$$

\section{Summary and outlook}

In this paper we have computed the universal part of the OPE coefficients of three heavy operators with no Lorentz spins. This contribution comes from the $\mathrm{AdS}_{2}$ part of the string $\sigma$-model, and has to be supplanted with the contribution of the $S^{5}$ part in order to obtain the full OPE coefficient of the relevant operators.

We employed the methods of Pohlmeyer reduction, which have been previously applied with great success to the case of null polygonal Wilson loops. It is interesting to notice that different aspects of the strong coupling physics of $\mathcal{N}=4 \mathrm{SYM}$ (gluon scattering amplitudes, anomalous dimensions through 2-point correlation functions, OPE coefficients) may be expressed by the same (modified) sinh-Gordon equation with all differences encoded in the analytical structure of the modification functions. Despite the similarities, the differences are significant, especially in the analytical structure and target-space reconstruction (as we are dealing with $\mathrm{AdS}_{2}$ instead of $\mathrm{AdS}_{3}$ ) which make the generalization nontrivial. As 
a cross-check of our results we made a comparison of our formulas with direct numerical solution of the modified sinh-Gordon equation.

Unfortunately, as the OPE coefficients of operators dual to semiclassical spinning strings have not been previously calculated, in general there are no independent results allowing for testing our final expression. Even in the case were we have some information, like specific BPS operators with large charges, our lack of knowledge of the $S^{5}$ contribution precludes a direct check.

However, there are two limits in which we may cross-check our formula. First of all, in the extremal case we find that the AdS contribution to the OPE coefficient does not have any semiclassical piece, as expected in this case. Secondly, when the anomalous dimensions are small ('medium'-type operators) our result is exactly equivalent to the Klose-McLoughlin formula obtained by a classical extremalization procedure of three point geodesics related to the three operators. It is reasonable to expect that such a point-like string/geodesic approximation to three point correlators is acceptable for not too 'heavy' operators, which do not generate an extended surface.

An additional consistency check is provided by the derivation of the correct CFT space-time dependence of the three-point correlators which arises from the regularized divergent part.

It would be interesting to perform a comparison with the case of heavy-heavy-light correlators. However it seems that such effects as leading order backreaction to the classical 2-point solution and corrections to vertex operators would have to be included in the heavyheavy-light calculations in order for the comparison to be made.

There are several directions in which our work could be further developed. Obviously, as we consider entirely the AdS contribution to the OPE coefficient, one has to perform an analogous analysis for the $S^{5}$ part in order to obtain the full OPE coefficient of the 'heavy' operators. We expect that the $S^{5}$ contribution will depend on the particularities of the states in questions and not only on their conformal dimensions. Moreover, all selection rules should show up from the $S^{5}$ contribution.

The $S^{5}$ part of the problem seems to be significantly more sophisticated for at least two reasons. The first reason is just technical, and should not pose too much problems. Namely, the simplest string solution rotates in $S^{3} \subset S^{5}$ which amounts to the reduction of the $\sigma$-model action to three dimensional case. Then, one should consider the corresponding Pohlmeyer reduction, again with a prescribed nonzero energy-momentum tensor. Since we deal with a higher dimensional target space, it leads to a more complicated (but still integrable) equations. A more serious problem is connected with the classical wavefunctions of the external states which must be taken into account as well. In particular it is not clear whether their contributions to the 3-point function would cancel out with their contributions to the 2-point functions when constructing a normalization independent OPE coefficient. Currently we lack an appropriate formulation with definite regularization prescription.

Further generalization would involve the computation of the OPE coefficients of operators carrying charges related to $\mathrm{AdS}_{5}$ momenta, but here again a consistent treatment of the wavefunctions would be needed. 
Finally, it would be very interesting to develop an analogous framework for higher point correlation functions and identify the general form of the key functional equations for the overlaps in this case.

\section{Acknowledgments}

We would like to thank Pedro Vieira and Amit Sever for initial collaboration, and Volodya Kazakov, Kostya Zarembo, Kolya Gromov, Tristan McLoughlin and Arkady Tseytlin for interesting discussions. RJ was supported by Polish science funds as a research project N N202 105136 (2009-2012). AW was supported by NCN grant 2011/01/B/ST2/00464.

\section{A Details of the numerical computation}

In order to solve numerically the modified sinh-Gordon equation with the boundary conditions relevant for 3-point correlation functions, we have to redefine the Pohlmeyer function since both the original version $\tilde{\gamma}$ and the modified one $\gamma$ are not suitable for numerics. $\tilde{\gamma}$ has logarithmic singularities at $w= \pm i a$, while $\tilde{\gamma}$ blows up at the punctures. A convenient choice which is finite everywhere is

$$
\gamma_{3}=\frac{1}{2} \tilde{\gamma}+\frac{1}{4} \log \left|w^{2}+a^{2}\right|^{2}-\frac{1}{4} \log \left(|w|^{4}+a^{2}\right)
$$

Another difficulty is to find a parametrization of the 3-punctured sphere which would allow us to use a rectangular grid of finite dimension. By symmetry, one can restrict oneself just to the upper quadrant $\operatorname{Re} w>0$ and $\operatorname{Im} w>0$. In this quadrant we used the following mapping of $w=x+i y$ :

$$
s=\frac{x}{x+1} \quad t=\frac{y}{y+1}
$$

and considered the unit square $s \in[0,1], t \in[0,1]$. The boundary conditions on the vertical edge $s=0$ follow from reflection symmetry, while those on the horizontal edge $t=0$ follow from the condition that $\tilde{\gamma}$ vanishes on the real line. This in turn is a consequence of the symmetry properties of the target space solution under a reflection $y \rightarrow-y$ which ensures that $\partial_{y} x=\partial_{y} z=0$ at $y=0$.

The upper and right edges of the square get mapped to the puncture at $w=\infty$, while $w=1$ is in the middle of the lower edge. We use Chebyshev spectral interpolation in the standard way in the $t$ coordinate and separately in two subintervals $s \in\left[0, \frac{1}{2}\right]$ and $s \in\left[\frac{1}{2}, 1\right]$ in order to allow for nonanalyticity at the puncture $w=1$ (here $(s, t)=\left(\frac{1}{2}, 0\right)$ ).

We use a Python interface to the PETSc library (petsc4py) to solve the spectrally discretized PDE. In order to get convergence we had to use an automatic differentiation package (ADOL-C with Python bindings pyadolc) to compute the Jacobian and use the LU linear solver from PETSc instead of the default iterative one. 


\section{B Evaluation of the integrals}

B.1 $\int_{C_{+}}\left(\int_{P_{+}}^{P} \omega\right) \eta=-i \pi \frac{1}{6}$

In the vicinity of the positive zero $w=i a$ the 1 -forms in the leading order read

$$
\omega \sim D \sqrt{w-i a} d w, \quad \eta \sim-\frac{1}{16 D} \frac{d w}{(w-i a)^{2}}
$$

where

$$
D=\frac{2 i a \Delta_{\infty}^{2}}{4\left(1+a^{2}\right)^{2}}
$$

Now, it is convenient to introduce a new variable $t^{2}=w-i a$. Since $p \in C_{+}$i.e., is located on an infinitesimal circle around $w=i a$, we parametrize it as $t=\epsilon e^{i \phi}$, where $\epsilon$ is an infinitesimal parameter and $\phi$ the angular variable. Then,

$$
\int_{P_{+}}^{P} \omega=2 \sqrt{D} \epsilon^{3} \int_{0}^{\phi} e^{3 i \phi^{\prime}} i d \phi^{\prime}+o\left(\epsilon^{3}\right)=\frac{2}{3} \sqrt{D} \epsilon^{3}\left(e^{3 i \phi}-1\right)+o\left(\epsilon^{3}\right)
$$

Now, we may insert this result into the contour integral

$$
\begin{aligned}
\int_{C_{+}}\left(\int_{P_{+}}^{P} \omega\right) \eta & =\int_{0}^{2 \pi} \frac{2}{3} \sqrt{D} \epsilon^{3}\left(e^{3 i \phi}-1\right) \frac{-1}{16 \sqrt{D}} \frac{1}{\epsilon^{5} e^{5 i \phi}} 2 \epsilon^{2} e^{2 i \phi} i d \phi+o\left(\epsilon^{0}\right) \\
& =\frac{-i}{12} \int_{0}^{2 \pi}\left(1-e^{-3 i \phi}\right) d \phi o\left(\epsilon^{0}\right)=\frac{-i \pi}{6}+o\left(\epsilon^{0}\right)
\end{aligned}
$$

\section{B.2 $\int_{C_{ \pm}} \eta=0$}

Here we prove that the integral of $\eta$ around a zero of $T(w)$ vanishes. Without loosing generality we assume $a=1$ which fixes the position of zero $w_{ \pm}= \pm i$. The cut is chosen to join the zeros. Now, consider an integral around $w_{+}$. The integral contour can be deformed in the following way

$$
\int_{C_{+}} \eta=\left(\int_{x,-1_{1}}+\int_{-1_{1}, \infty_{1}}+\int_{\infty_{1}, 1_{1}}+\int_{1_{1}, x}\right) \eta+\left(\int_{x,-1_{2}}+\int_{-1_{2}, \infty_{2}}+\int_{\infty_{2}, 1_{2}}+\int_{1_{2}, x}\right) \eta
$$

where the subscript denotes the Riemann sheet of the doouble cover $\widetilde{\Sigma}$ and $x$ a fixed point on the cut. Now consider a single pair of integrals of the form

$$
\int_{-1_{1}, \infty_{1}} \eta+\int_{-1_{2}, \infty_{2}} \eta
$$

Since $\eta$ is proportional to $\sqrt{\bar{T}}$, it differs on the two sheets just by a relative sign. So the sum of the two integrals is zero. Repeating this argument we arrive at

$$
\int_{C_{+}} \eta=0
$$




\section{The zero mode parts}

In general, the zero-mode constants are given by the following expression

$$
M_{12}=\lim _{w_{1}^{\prime} \rightarrow w_{1}} \lim _{w_{2}^{\prime} \rightarrow w_{2}} \int_{w_{1}^{\prime}}^{w_{2}^{\prime}} \frac{1}{2} \sqrt{T(w)} d w+\frac{\Delta_{1}}{4} \ln \left(w_{1}-w_{1}^{\prime}\right)+\frac{\Delta_{2}}{4} \ln \left(w_{2}-w_{2}^{\prime}\right)
$$

where $w_{1}, w_{2}$ are the end points for the WKB trajectory (poles of $T(w)$ ) with corresponding anomalous dimensions $\Delta_{1}, \Delta_{2}$. In our case

$$
\begin{aligned}
\int \sqrt{T(w)} d w & =\frac{\Delta_{\infty}}{2} \int \frac{\sqrt{w^{2}+a^{2}}}{1-w^{2}} d w= \\
& =\frac{\Delta_{\infty}}{4}\left[\sqrt{1+a^{2}} \ln \left(\frac{1+w}{1-w} \cdot \frac{a^{2}+w+\sqrt{1+a^{2}} \sqrt{a^{2}+w^{2}}}{a^{2}-w+\sqrt{1+a^{2}} \sqrt{a^{2}+w^{2}}}\right)-2 \ln \left(w+\sqrt{a^{2}+w^{2}}\right)\right] \\
& =\frac{\Delta}{2} \cdot \ln \left(\frac{1+w}{1-w} \cdot \frac{a^{2}+w+\sqrt{1+a^{2}} \sqrt{a^{2}+w^{2}}}{a^{2}-w+\sqrt{1+a^{2}} \sqrt{a^{2}+w^{2}}}\right)-\frac{\Delta_{\infty}}{2} \cdot \ln \left(w+\sqrt{a^{2}+w^{2}}\right) \quad(\text { C. } 2)
\end{aligned}
$$

One may easily verify that the first term gives the expected asymptotics at $w=1$ and $w=-1$ (with the correct $\frac{\Delta}{2}$ factor due to the fact that $\sqrt{1+a^{2}}=\frac{2 \Delta}{\Delta_{\infty}}$ ), while the second term is responsible for the asymptotics at $w=\infty$.

For the punctures $w_{1}=-1, w_{2}=1$ we get a cancellation of singularities in (C.1) and find the following finite result

$$
M_{-1,1}=\frac{\Delta}{2} \ln \frac{8 \Delta^{2}}{4 \Delta^{2}-\Delta_{\infty}^{2}}-\frac{\Delta_{\infty}}{4} \ln \frac{2 \Delta+\Delta_{\infty}}{2 \Delta-\Delta_{\infty}}+\frac{\Delta}{4} i \pi
$$

In order to calculate $M_{\infty, 1}$ it is convenient to perform a change of variables $w \rightarrow \frac{1}{z}$. Then $M_{\infty, 1}=\tilde{M}_{0,1}$, where

$$
\tilde{M}_{0,1}=\lim _{z_{1}^{\prime} \rightarrow z_{1}=0} \lim _{z_{2}^{\prime} \rightarrow z_{2}=1} \int_{z_{1}^{\prime}}^{z_{2}^{\prime}} \frac{1}{2} \sqrt{T(1 / z)} \frac{d z}{-z^{2}}+\frac{\Delta_{\infty}}{4} \ln \left(z_{1}-z_{1}^{\prime}\right)+\frac{\Delta}{4} \ln \left(z_{2}-z_{2}^{\prime}\right)
$$

Therefore,

$$
\begin{aligned}
\tilde{M}_{0,1}= & \left.\lim _{z_{1}^{\prime} \rightarrow z_{1}=0} \lim _{z_{2}^{\prime} \rightarrow z_{2}=1} \frac{\Delta}{4} \cdot \ln \left(\frac{1+\frac{1}{z}}{1-\frac{1}{z}} \cdot \frac{a^{2}+\frac{1}{z}+\sqrt{1+a^{2}} \sqrt{a^{2}+\frac{1}{z^{2}}}}{a^{2}-\frac{1}{z}+\sqrt{1+a^{2}} \sqrt{a^{2}+\frac{1}{z^{2}}}}\right)\right|_{z_{1}^{\prime}} ^{z_{2}^{\prime}} \\
& -\left.\frac{\Delta_{\infty}}{4} \cdot \ln \left(\frac{1}{z}+\sqrt{a^{2}+\frac{1}{z^{2}}}\right)\right|_{z_{1}^{\prime}} ^{z_{2}^{\prime}}+\frac{\Delta_{\infty}}{4} \ln \left(-z_{1}^{\prime}\right)+\frac{\Delta}{4} \ln \left(z_{2}-z_{2}^{\prime}\right)
\end{aligned}
$$

The singular part at $z_{1}^{\prime} \rightarrow z_{1}=0$ is

$$
\frac{\Delta_{\infty}}{4} \cdot \ln \left(\frac{1}{z_{1}^{\prime}}+\sqrt{a^{2}+\frac{1}{z_{1}^{\prime 2}}}\right)+\frac{\Delta_{\infty}}{4} \ln \left(-z_{1}^{\prime}\right)=\frac{\Delta_{\infty}}{4} \cdot \ln \left(\frac{2}{z_{1}^{\prime}}\right)+\frac{\Delta_{\infty}}{4} \ln \left(-z_{1}^{\prime}\right)=\frac{\Delta_{\infty}}{4}(\ln 2+i \pi)
$$


while the finite part at $z_{1}^{\prime} \rightarrow z_{1}=0$ reads

$$
\lim _{z_{1}^{\prime} \rightarrow z_{1}=0}-\frac{\Delta}{4} \cdot \ln \left(\frac{1+\frac{1}{z_{1}^{\prime}}}{1-\frac{1}{z_{1}^{\prime}}} \cdot \frac{a^{2}+\frac{1}{z_{1}^{\prime}}+\sqrt{1+a^{2}} \sqrt{a^{2}+\frac{1}{z_{1}^{\prime 2}}}}{a^{2}-\frac{1}{z_{1}^{\prime}}+\sqrt{1+a^{2}} \sqrt{a^{2}+\frac{1}{z_{1}^{\prime 2}}}}\right)=-\frac{\Delta}{4} \ln \left(\frac{1+\sqrt{1+a^{2}}}{-1+\sqrt{1+a^{2}}}\right)-\frac{\Delta}{4} \ln (-1)
$$

The part at $z_{1}^{\prime} \rightarrow z_{1}=1$ is

$$
\begin{gathered}
\lim _{z_{2}^{\prime} \rightarrow z_{2}=1} \frac{\Delta}{4} \cdot \ln \left(\frac{1+\frac{1}{z_{2}^{\prime}}}{1-\frac{1}{z_{2}^{\prime}}} \cdot \frac{a^{2}+\frac{1}{z_{2}^{\prime}}+\sqrt{1+a^{2}} \sqrt{a^{2}+\frac{1}{z_{2}^{\prime 2}}}}{a^{2}-\frac{1}{z_{2}^{\prime}}+\sqrt{1+a^{2}} \sqrt{a^{2}+\frac{1}{z_{2}^{\prime 2}}}}\right)-\frac{\Delta_{\infty}}{4} \cdot \ln \left(\frac{1}{z_{2}^{\prime}}+\sqrt{a^{2}+\frac{1}{z_{2}^{\prime 2}}}\right)+\frac{\Delta}{4} \ln \left(1-z_{2}^{\prime}\right) \\
=\lim _{z_{2}^{\prime} \rightarrow z_{2}=1} \frac{\Delta}{4} \cdot \ln \left(\frac{z_{2}^{\prime}+1}{z_{2}^{\prime}-1} \cdot \frac{a^{2}+\frac{1}{z_{2}^{\prime}}+\sqrt{1+a^{2}} \sqrt{a^{2}+\frac{1}{z_{2}^{\prime 2}}}}{a^{2}-\frac{1}{z_{2}^{\prime}}+\sqrt{1+a^{2}} \sqrt{a^{2}+\frac{1}{z_{2}^{\prime 2}}}}\right) \\
-\frac{\Delta_{\infty}}{4} \cdot \ln \left(\frac{1}{z_{2}^{\prime}}+\sqrt{a^{2}+\frac{1}{z_{2}^{\prime 2}}}\right)+\frac{\Delta}{4} \ln \left(z_{2}^{\prime}-1\right)+\frac{\Delta}{4} i \pi
\end{gathered}
$$

The singular parts $\sim \ln \left(z_{2}^{\prime}-1\right)$ again cancel and we are left with

$$
\frac{\Delta}{4} \ln \frac{2\left(a^{2}+1\right)}{a^{2}}-\frac{\Delta_{\infty}}{4} \cdot \ln \left(1+\sqrt{a^{2}+1}\right)+\frac{\Delta}{4} i \pi
$$

Finally we get

$$
\tilde{M}_{0,1}=\frac{\Delta_{\infty}}{4}(\ln 2+i \pi)-\frac{\Delta}{4} \ln \left(\frac{1+\sqrt{1+a^{2}}}{-1+\sqrt{1+a^{2}}}\right)+\frac{\Delta}{4} \ln \frac{2\left(a^{2}+1\right)}{a^{2}}-\frac{\Delta_{\infty}}{4} \cdot \ln \left(1+\sqrt{a^{2}+1}\right)
$$

which may be simplified to (recall that $M_{\infty, 1}=\tilde{M}_{0,1}$ )

$$
M_{\infty, 1}=\frac{\Delta_{\infty}}{4}(\ln 2+i \pi)+\frac{\Delta}{4} \ln 2-\frac{\Delta_{\infty}}{4} \cdot \ln \left(\frac{\Delta_{\infty}+2 \Delta}{\Delta_{\infty}}\right)-\frac{\Delta}{2} \ln \left(\frac{2 \Delta+\Delta_{\infty}}{2 \Delta}\right)
$$

\section{Small $a$ asymptotics of $\tilde{h}(a)$}

We divide the integral into two parts

$$
\begin{aligned}
\tilde{h}(a) & =\frac{1}{2 \pi} \int_{-\infty}^{\infty} \frac{\sinh ^{2} \theta}{\cosh \theta} \ln \left(1-e^{-a \pi \cosh \theta}\right) \\
& =\frac{1}{\pi}\left[\int_{0}^{\infty} \cosh \theta \ln \left(1-e^{-a \pi \cosh \theta}\right)-\int_{0}^{\infty} \frac{1}{\cosh \theta} \ln \left(1-e^{-a \pi \cosh \theta}\right)\right]
\end{aligned}
$$

The first integral was calculated in [55] and has the following asymptotics for small $a$

$$
\int_{0}^{\infty} \cosh \theta \ln \left(1-e^{-a \pi \cosh \theta}\right)=-\frac{\pi}{6 a}+o(1)
$$

In order to compute the second integral we notice that

$$
\ln \left(1-e^{-a \pi \cosh \theta}\right)=\ln (a \cosh \theta)+o(1)
$$


Then, the integral is finite in this limit and reads

$$
\begin{aligned}
\int_{0}^{\infty} \frac{1}{\cosh \theta} \ln \left(1-e^{-a \pi \cosh \theta}\right) & =\int_{0}^{\infty} \frac{1}{\cosh \theta} \ln (a \cosh \theta)+o(1) \\
& =\frac{\pi}{2} \ln a+o(1)
\end{aligned}
$$

Inserting these results to the integral we get at the leading order

$$
\tilde{h}=-\frac{\pi}{6 a}-\frac{1}{2} \ln a
$$

Open Access. This article is distributed under the terms of the Creative Commons Attribution Noncommercial License which permits any noncommercial use, distribution, and reproduction in any medium, provided the original author(s) and source are credited.

\section{References}

[1] I. Bena, J. Polchinski and R. Roiban, Hidden symmetries of the $A d S_{5} \times S^{5}$ superstring, Phys. Rev. D 69 (2004) 046002 [hep-th/0305116] [INSPIRE].

[2] J.A. Minahan and K. Zarembo, The Bethe ansatz for $N=4$ super Yang-Mills, JHEP 03 (2003) 013 [hep-th/0212208] [INSPIRE].

[3] V.A. Kazakov, A. Marshakov, J.A. Minahan and K. Zarembo, Classical/quantum integrability in AdS/CFT, JHEP 05 (2004) 024 [hep-th/0402207] [INSPIRE].

[4] N. Beisert, V. Dippel and M. Staudacher, A novel long range spin chain and planar $N=4$ super Yang-Mills, JHEP 07 (2004) 075 [hep-th/0405001] [INSPIRE].

[5] G. Arutyunov, S. Frolov and M. Staudacher, Bethe ansatz for quantum strings, JHEP 10 (2004) 016 [hep-th/0406256] [INSPIRE].

[6] N. Beisert and M. Staudacher, Long-range PSU $(2,2 \mid 4)$ Bethe ansätze for gauge theory and strings, Nucl. Phys. B 727 (2005) 1 [hep-th/0504190] [INSPIRE].

[7] N. Beisert, The SU(2|2) dynamic S-matrix, Adv. Theor. Math. Phys. 12 (2008) 945 [hep-th/0511082] [INSPIRE].

[8] R.A. Janik, The $A d S_{5} \times S^{5}$ superstring worldsheet $S$-matrix and crossing symmetry, Phys. Rev. D 73 (2006) 086006 [hep-th/0603038] [INSPIRE].

[9] N. Beisert, R. Hernandez and E. Lopez, A crossing-symmetric phase for $A d S_{5} \times S^{5}$ strings, JHEP 11 (2006) 070 [hep-th/0609044] [INSPIRE].

[10] N. Beisert, B. Eden and M. Staudacher, Transcendentality and crossing, J. Stat. Mech. (2007) P01021 [hep-th/0610251] [inSPIRE].

[11] G. Arutyunov and S. Frolov, String hypothesis for the $A d S_{5} \times S^{5}$ mirror, JHEP 03 (2009) 152 [arXiv:0901.1417] [inSPIRE].

[12] N. Gromov, V. Kazakov and P. Vieira, Exact spectrum of anomalous dimensions of planar $N=4$ supersymmetric Yang-Mills theory, Phys. Rev. Lett. 103 (2009) 131601 [arXiv: 0901.3753] [INSPIRE].

[13] D. Bombardelli, D. Fioravanti and R. Tateo, Thermodynamic Bethe ansatz for planar AdS/CFT: a proposal, J. Phys. A 42 (2009) 375401 [arXiv:0902.3930] [InSPIRE]. 
[14] N. Gromov, V. Kazakov, A. Kozak and P. Vieira, Exact spectrum of anomalous dimensions of planar $N=4$ supersymmetric Yang-Mills theory: TBA and excited states, Lett. Math. Phys. 91 (2010) 265 [arXiv:0902.4458] [INSPIRE].

[15] G. Arutyunov and S. Frolov, Thermodynamic Bethe ansatz for the $A d S_{5} \times S^{5}$ mirror model, JHEP 05 (2009) 068 [arXiv:0903.0141] [INSPIRE].

[16] J.M. Maldacena, The large- $N$ limit of superconformal field theories and supergravity, Int. J. Theor. Phys. 38 (1999) 1113 [Adv. Theor. Math. Phys. 2 (1998) 231] [hep-th/9711200] [INSPIRE].

[17] S.S. Gubser, I.R. Klebanov and A.M. Polyakov, Gauge theory correlators from noncritical string theory, Phys. Lett. B 428 (1998) 105 [hep-th/9802109] [INSPIRE].

[18] E. Witten, Anti-de Sitter space and holography, Adv. Theor. Math. Phys. 2 (1998) 253 [hep-th/9802150] [INSPIRE].

[19] S.S. Gubser, I.R. Klebanov and A.M. Polyakov, A semiclassical limit of the gauge/string correspondence, Nucl. Phys. B 636 (2002) 99 [hep-th/0204051] [INSPIRE].

[20] S. Frolov and A.A. Tseytlin, Multispin string solutions in $A d S_{5} \times S^{5}$, Nucl. Phys. B 668 (2003) 77 [hep-th/0304255] [INSPIRE].

[21] A.A. Tseytlin, Review of AdS/CFT integrability, chapter II.1: classical $A d S_{5} \times S^{5}$ string solutions, arXiv: 1012.3986 [INSPIRE].

[22] D.Z. Freedman, S.D. Mathur, A. Matusis and L. Rastelli, Correlation functions in the CFT $(d) / A d S(d+1)$ correspondence, Nucl. Phys. B 546 (1999) 96 [hep-th/9804058] [INSPIRE].

[23] G. Arutyunov and S. Frolov, Some cubic couplings in type IIB supergravity on $A d S_{5} \times S^{5}$ and three point functions in SYM(4) at large-N, Phys. Rev. D 61 (2000) 064009 [hep-th/9907085] [INSPIRE].

[24] R.A. Janik, P. Surowka and A. Wereszczynski, On correlation functions of operators dual to classical spinning string states, JHEP 05 (2010) 030 [arXiv:1002.4613] [INSPIRE].

[25] A. Tsuji, Holography of Wilson loop correlator and spinning strings, Prog. Theor. Phys. 117 (2007) 557 [hep-th/0606030] [INSPIRE].

[26] E.I. Buchbinder and A.A. Tseytlin, On semiclassical approximation for correlators of closed string vertex operators in AdS/CFT, JHEP 08 (2010) 057 [arXiv: 1005.4516] [INSPIRE].

[27] K. Zarembo, Holographic three-point functions of semiclassical states, JHEP 09 (2010) 030 [arXiv: 1008.1059] [INSPIRE].

[28] M.S. Costa, R. Monteiro, J.E. Santos and D. Zoakos, On three-point correlation functions in the gauge/gravity duality, JHEP 11 (2010) 141 [arXiv:1008.1070] [INSPIRE].

[29] R. Roiban and A.A. Tseytlin, On semiclassical computation of 3-point functions of closed string vertex operators in $A d S_{5} \times S^{5}$, Phys. Rev. D 82 (2010) 106011 [arXiv:1008.4921] [INSPIRE].

[30] R. Hernandez, Three-point correlation functions from semiclassical circular strings, J. Phys. A 44 (2011) 085403 [arXiv: 1011. 0408] [inSPIRE].

[31] S. Ryang, Correlators of vertex operators for circular strings with winding numbers in $A d S_{5} \times S^{5}, J H E P 01$ (2011) 092 [arXiv:1011.3573] [INSPIRE]. 
[32] G. Georgiou, Two and three-point correlators of operators dual to folded string solutions at strong coupling, JHEP 02 (2011) 046 [arXiv:1011.5181] [INSPIRE].

[33] J.G. Russo and A.A. Tseytlin, Large spin expansion of semiclassical 3-point correlators in $A d S_{5} \times S^{5}, J H E P 02$ (2011) 029 [arXiv: 1012.2760] [INSPIRE].

[34] C. Park and B.-H. Lee, Correlation functions of magnon and spike, Phys. Rev. D 83 (2011) 126004 [arXiv: 1012.3293] [inSPIRE].

[35] E.I. Buchbinder and A.A. Tseytlin, Semiclassical four-point functions in $A d S_{5} \times S^{5}$, JHEP 02 (2011) 072 [arXiv: 1012.3740] [INSPIRE].

[36] D. Bak, B. Chen and J.-B. Wu, Holographic correlation functions for open strings and branes, JHEP 06 (2011) 014 [arXiv:1103.2024] [INSPIRE].

[37] A. Bissi, C. Kristjansen, D. Young and K. Zoubos, Holographic three-point functions of giant gravitons, JHEP 06 (2011) 085 [arXiv: 1103.4079] [INSPIRE].

[38] D. Arnaudov, R.C. Rashkov and T. Vetsov, Three and four-point correlators of operators dual to folded string solutions in $A d S_{5} \times S^{5}$, Int. J. Mod. Phys. A 26 (2011) 3403 [arXiv:1103.6145] [INSPIRE].

[39] R. Hernandez, Three-point correlators for giant magnons, JHEP 05 (2011) 123 [arXiv:1104.1160] [INSPIRE].

[40] C. Ahn and P. Bozhilov, Three-point correlation functions of giant magnons with finite size, Phys. Lett. B 702 (2011) 286 [arXiv:1105.3084] [InSPIRE].

[41] L.F. Alday and A.A. Tseytlin, On strong-coupling correlation functions of circular Wilson loops and local operators, J. Phys. A 44 (2011) 395401 [arXiv:1105.1537] [INSPIRE].

[42] L.F. Alday, E.I. Buchbinder and A.A. Tseytlin, Correlation function of null polygonal Wilson loops with local operators, JHEP 09 (2011) 034 [arXiv: 1107.5702] [INSPIRE].

[43] T. Klose and T. McLoughlin, A light-cone approach to three-point functions in $A d S_{5} \times S^{5}$, arXiv: 1106.0495 [INSPIRE].

[44] J. Escobedo, N. Gromov, A. Sever and P. Vieira, Tailoring three-point functions and integrability, JHEP 09 (2011) 028 [arXiv: 1012.2475] [INSPIRE].

[45] J. Escobedo, N. Gromov, A. Sever and P. Vieira, Tailoring three-point functions and integrability II. Weak/strong coupling match, JHEP 09 (2011) 029 [arXiv:1104.5501] [INSPIRE].

[46] A.A. Tseytlin, On semiclassical approximation and spinning string vertex operators in $A d S_{5} \times S^{5}$, Nucl. Phys. B $664(2003) 247$ [hep-th/0304139] [INSPIRE].

[47] E.I. Buchbinder, Energy-spin trajectories in $A d S_{5} \times S^{5}$ from semiclassical vertex operators, JHEP 04 (2010) 107 [arXiv: 1002.1716] [INSPIRE].

[48] K. Pohlmeyer, Integrable Hamiltonian systems and interactions through quadratic constraints, Commun. Math. Phys. 46 (1976) 207 [INSPIRE].

[49] H.J. de Vega and N.G. Sanchez, Exact integrability of strings in D-dimensional de Sitter space-time, Phys. Rev. D 47 (1993) 3394 [INSPIRE].

[50] L.F. Alday and J.M. Maldacena, Null polygonal Wilson loops and minimal surfaces in anti-de-Sitter space, JHEP 11 (2009) 082 [arXiv:0904.0663] [INSPIRE]. 
[51] L.F. Alday, J.M. Maldacena, A. Sever and P. Vieira, Y-system for scattering amplitudes, J. Phys. A 43 (2010) 485401 [arXiv:1002.2459] [InSPIRE].

[52] J.M. Maldacena and A. Zhiboedov, Form factors at strong coupling via a Y-system, JHEP 11 (2010) 104 [arXiv: 1009.1139] [inSPIRE].

[53] D. Gaiotto, G.W. Moore and A. Neitzke, Wall-crossing, Hitchin systems and the WKB approximation, arXiv:0907.3987 [INSPIRE].

[54] A.B. Zamolodchikov, Painleve III and 2-d polymers, Nucl. Phys. B 432 (1994) 427 [hep-th/9409108] [INSPIRE].

[55] N. Gromov, D. Serban, I. Shenderovich and D. Volin, Quantum folded string and integrability: from finite size effects to Konishi dimension, JHEP 08 (2011) 046 [arXiv:1102.1040] [INSPIRE]. 